\title{
The complementary RANS equations for the simulation of viscous flows
}

\author{
Kunho Kim, Ana I. Sirviente ${ }^{*, \dagger}$ and Robert F. Beck \\ Department of Naval Architecture and Marine Engineering, The University of Michigan, \\ Ann Arbor, MI 48109, U.S.A.
}

\begin{abstract}
SUMMARY
A complementary set of Reynolds-averaged Navier-Stokes (RANS) equations has been developed for steady incompressible, turbulent flows. The method is based on the Helmholtz decomposition of the velocity vector field into a viscous and a potential components. In the complementary RANS solver a potential solution coexists with a viscous solution with the purpose of contributing to a fastest decay of the viscous solution in the far field. The proposed complementary RANS equations have been validated for steady laminar and turbulent flows. The computational results show that the complementary RANS solver is able to produce less grid-dependent solutions than a conventional RANS solver. Copyright (C) 2005 John Wiley \& Sons, Ltd.
\end{abstract}

KEY WORDS: complementary RANS equations; RANS solver; viscous-inviscid coupling; Helmholtz decomposition

\section{INTRODUCTION}

Viscous effects play an important role in a variety of hydrodynamic problems. To account for the viscous effects, researchers have investigated the interactions between inviscid and viscous flows since Prandtl's boundary layer theory in the early 1900s. Prandtl [1] assumed viscous effects are confined to a thin layer and derived the boundary layer theory. In early aeronautics, researchers used this thin layer approximation to improve inviscid potential solutions by adding the displacement thickness to the body shape or by using the transpiration velocity concept of Lighthill [2]. Lighthill proposed four alternative treatments of displacement thickness for two- and three-dimensional flows, which are called the methods of flow reduction, equivalent sources, velocity comparison, and mean vorticity. A detailed review of the various viscous-

\footnotetext{
${ }^{*}$ Correspondence to: Ana I. Sirviente, Department of Naval Architecture and Marine Engineering, The University of Michigan, Ann Arbor, MI 48109, U.S.A.

†E-mail: asirv@engin.umich.edu

Contract/grant sponsor: Office of Naval Research; contract/grant number: N00014-01-2-0008
}

Received 25 March 2004

Copyright (c) 2005 John Wiley \& Sons, Ltd.

Revised 27 October 2004 Accepted 27 October 2004 
inviscid interaction techniques developed mostly for aerodynamic applications can be found in Lock and Williams [3].

In principle, the viscous effects can be captured by the full Navier-Stokes equations for the entire fluid domain. Among others, Miyata et al. [4], Farmer et al. [5] and Tahara and Stern [6] have investigated ship hydrodynamic problems with free surfaces by using full Reynoldsaveraged Navier-Stokes (RANS) equations. Miyata et al. [4] developed a finite-volume method employing an explicit time marching procedure. Farmer et al. [5] used a multi-grid method to accelerate the computations. Tahara and Stern [6] utilized a finite-analytic method with the Baldwin-Lomax turbulence model. These calculations, however, require a significant amount of computational resources. Since the viscous effects are confined in a thin region around the body and in the wake, a combination of a viscous solver and a relatively simpler potential solver can provide considerable savings in computational time and memory requirement.

Numerical techniques involving the coupling of potential/RANS solvers have been proposed in the past for ship hydrodynamic problems. Among others, Stern et al. [7], Tahara et al. [8], Villeger and Alessandrini [9], Chen et al. [10], Campana et al. [11, 12], Chen and Lee [13, 14], Dommermuth et al. [15] and Ferrant et al. [16] have all studied viscous-inviscid interactions. Stern et al. [7] employed a displacement body concept to solve the partially parabolic RANS equations. They compared the full RANS solutions in a large domain with interactive viscousinviscid solutions. Although both gave satisfactory results, it was concluded that the interactive approach is computationally more efficient. Similar techniques were utilized by Tahara et al. [8] for the Wigley hull. They used the SPLASH potential code along with a RANS equations solver based on the finite analytic method. Villeger and Alessandrini [9] solved the boundary layer equations in combination with a potential solution and the concept of transpiration velocity.

Although these studies produced satisfactory results, the displacement thickness is sensitive to small velocity changes in the outer parts of the viscous layer. Moreover, the displacement thickness concept becomes questionable as the boundary layer thickens or if flow separation takes place. To overcome this problem, Chen et al. [10] proposed a velocity/pressure matching scheme. They solved the RANS equations based on the finite analytic method for the viscous near field, which was matched with a potential solution. Two potential codes were employed; LAMP (large-amplitude motion program) and SLAW (ship lift and wave). LAMP uses a time-domain Green function approach and SLAW uses a Dawson type steady ship wave panel method. Computations were performed for a Series 60 bare hull $\left(C_{\mathrm{b}}=0.6\right)$, where $C_{\mathrm{b}}$ is the block coefficient. Campana et al. $[11,12]$ utilized a similar matching idea between a RANS equations finite volume scheme and a linear potential code. They chose the Dawson model for the free-surface external flow. Chen and Lee [13] employed a nonlinear potential code combined with a RANS code using the finite analytic method for a submerged foil with and without the presence of free surface waves. An extension to a surface piercing body was exploited in Chen and Lee [14]. Although it is clear from the point of view of computational time that the potential/RANS matching method is advantageous over full viscous RANS computations, this approach still involves a fairly large domain for the computation of the viscous effects.

Thus far, researchers have used a potential solver separately either by using potential solutions as initial conditions or by matching potential solutions to viscous solutions in separate regions. However, little has been done to directly couple the use of potential solutions with viscous solutions. 

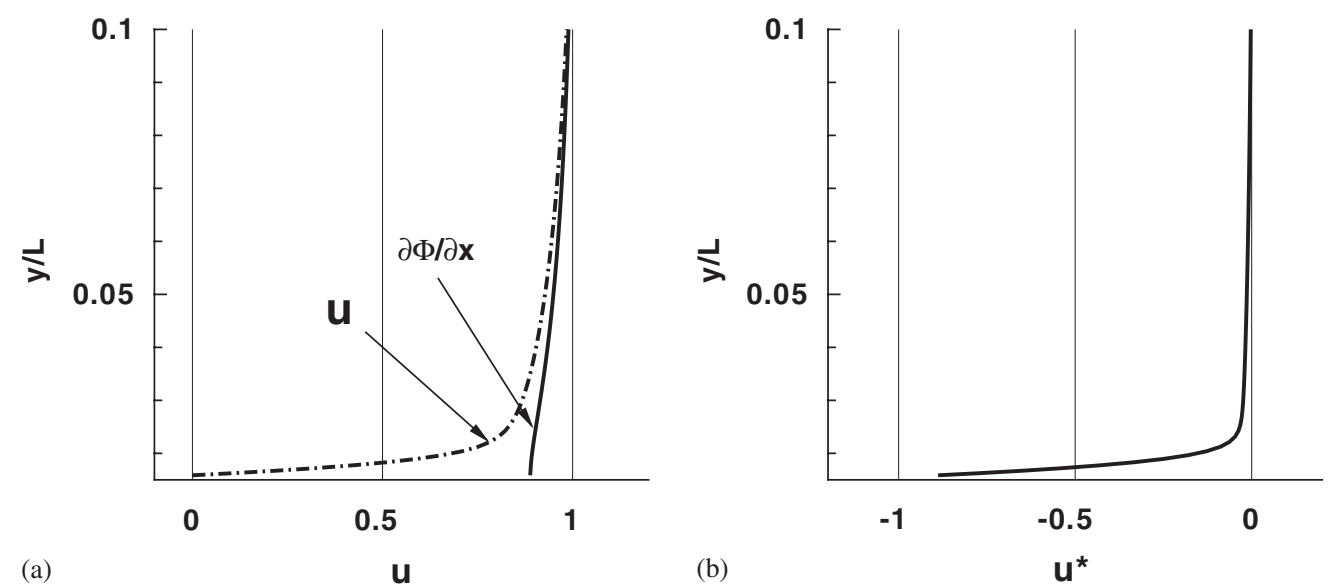

Figure 1. Horizontal velocities from a conventional solver $(u)$, a potential solver $(\partial \Phi / \partial x)$ and a complementary solver $\left(u^{*}\right)$ : (a) $u$ and $\partial \Phi / \partial x$; and (b) complementary $u^{*}$.

Recently, Dommermuth et al. [15] employed a decomposition to solve the contact line problems in bow waves. They decomposed the flow into an irrotational portion and a vortical portion. The vortical portion was used to enforce the no-slip boundary condition on the hull and the irrotational portion was used to impose the free-surface boundary conditions. Ferrant et al. [16] also proposed a potential/RANSE (RANS equations) formulation by using a decomposition of velocity and pressure into an incident part and a diffracted part. In their method, the Euler equations are solved for the incident part and modified RANS equations, socalled, SWENS (spectral wave explicit Navier-Stokes) equations are solved for the diffraction part in a submerged square cylinder problem with a free surface.

In the present study, the so-called complementary RANS equations are derived and proposed as an alternative to the conventional RANS equations. The complementary RANS equations yield the difference between the viscous flow solution found by the conventional RANS equations and an arbitrary potential flow solution. Consequently the new set of equations can be solved to obtain the corresponding complementary velocity field $u_{i}^{*}\left(u_{i}^{*}=u_{i}-u_{p_{i}}\right)$, where $u_{i}$ is the solution of the conventional RANS equations, and $u_{p_{i}}$ is the arbitrary potential flow solution.

Figure 1 shows typical horizontal velocity profiles on a body surface in a two-dimensional external flow problem. In Figure 1(a), the dashed line is a velocity profile from a RANS solver and the solid line is a velocity profile from a potential solver. Since the potential solver is not able to enforce the no-slip condition on the wall, there is a slip in the velocity at the wall. The gap between the viscous solution and the potential solution is largest on the body and decreases as the flow moves from the body. And finally in the far field, the potential solution is identical to the viscous solution. If the velocity, $u_{i}$, is decomposed into a potential part, $u_{p_{i}}$, and the remaining part, $u_{i}^{*}$, as mentioned earlier, the $u_{i}^{*}$ solution will be as shown in Figure 1(b). Since $u_{i}^{*}$ is basically the difference between the viscous solution and the potential solution, it is largest on the body and approaches zero as the flow moves away from the body. Given a potential solution, if the velocity in the governing equations 
is replaced with a new variable for the remaining part, $u_{i}^{*}$, then the resulting solutions will approach zero in the far field, with the proper choice of the potential solution. The best choice of the potential solution will be the one that forces $u_{i}^{*}$ to go to zero the fastest reducing to a minimum the region of the domain where viscous effects are relevant. With these equations, it might be possible to develop completely different methodologies to numerically solve external flow problems that would possibly be more computationally efficient than the current RANS solvers applied to the complete domain. This is the motivation for the present study.

In this study, the complementary RANS equations are derived and validated for steady laminar and turbulent flow problems such as flows in a square duct, over a flat plate, over a NACA 0010 and over a NACA 0012 airfoil. The overview of mathematical formulation supporting the derivation of the complementary RANS equations is presented in the following section, which is followed by the numerical methods employed in the study. A discussion of the computational results obtained for the various flows simulated follows in Section 4. The advantages and disadvantages of the proposed scheme are also discussed in comparison to the performance of a conventional RANS solver.

\section{MATHEMATICAL FORMULATION}

In this section the complementary RANS equations are derived along with the corresponding modified $k-\varepsilon$ turbulence closure. A Cartesian inertial co-ordinate system is employed with the positive $z$-axis vertically upward for three-dimensional problems. For two-dimensional problems, the vertical co-ordinate becomes $y$.

Using the Helmholtz decomposition [17], a velocity vector, $u_{i}$, can be decomposed into a rotational, $u_{i}^{*}$, and an irrotational part, $u_{p_{i}}$. The potential velocity vector, $u_{p_{i}}$, can consequently be expressed as the gradient of a velocity potential, $\Phi$, such that

$$
u_{p_{i}}=\frac{\partial \Phi}{\partial x_{i}}
$$

where $\Phi$, for an incompressible fluid, satisfies the Laplace equation as follows:

$$
\nabla^{2} \Phi=0
$$

By definition, $\Phi$ is irrotational (i.e. vorticity-free), thus all the vorticity is included in $u_{i}^{*}$. The velocity vector, $u_{i}$, can consequently be defined as

$$
u_{i}=u_{i}^{*}+u_{p_{i}}
$$

and $u_{i}$ must satisfy the continuity equation and Navier-Stokes equations for incompressible viscous flows. Substituting Equation (3) into the continuity equation and using the Laplace equation, it can be shown that the complementary velocity vector must satisfy the divergencefree condition as follows:

$$
\frac{\partial u_{i}^{*}}{\partial x_{i}}=0
$$

It should be noted that the velocity decomposition is not unique even with the divergencefree requirement for the rotational part. Consequently for any chosen velocity potential, 
a complementary velocity can be obtained by subtracting the potential velocity vector from the total velocity vector.

The velocity vector $u_{i}$ must also satisfy the Navier-Stokes equations as mentioned earlier. The Navier-Stokes or the momentum equations for incompressible viscous flows are given by

$$
\frac{\partial u_{i}}{\partial t}+u_{j} \frac{\partial u_{i}}{\partial x_{j}}=-\frac{\partial p}{\partial x_{i}}+\frac{1}{R e} \nabla^{2} u_{i}+\frac{1}{F r^{2}} \delta_{i 3}
$$

where $p$ is the pressure, $R e=\rho U_{0} L / \mu$ is the Reynolds number ( $\rho$ is fluid density, $U_{0}$ is the free stream velocity, $L$ is length scale, and $\mu$ is viscosity), $F r=U_{0} / \sqrt{g L}$ is the Froude number ( $g$ is the gravitational acceleration), and $\delta_{i j}$ is the Kronecker delta.

Substituting the velocity decomposition (Equation (3)) into the momentum equations (Equation (5)) gives

$$
\frac{\partial\left(u_{p_{i}}+u_{i}^{*}\right)}{\partial t}+\left(u_{p_{j}}+u_{j}^{*}\right) \frac{\partial\left(u_{p_{i}}+u_{i}^{*}\right)}{\partial x_{j}}=-\frac{\partial p}{\partial x_{i}}+\frac{1}{R e} \nabla^{2}\left(u_{p_{i}}+u_{i}^{*}\right)+\frac{1}{F r^{2}} \delta_{i 3}
$$

After some mathematical manipulation, Equation (6) can be written as

$$
\frac{\partial u_{p_{i}}}{\partial t}+\frac{\partial u_{i}^{*}}{\partial t}+\left(u_{p_{j}}+u_{j}^{*}\right) \frac{\partial u_{i}^{*}}{\partial x_{j}}+\left(u_{p_{j}}+u_{j}^{*}\right) \frac{\partial u_{p_{i}}}{\partial x_{j}}=-\frac{\partial p}{\partial x_{i}}+\frac{1}{R e} \nabla^{2} u_{i}^{*}+\frac{1}{F r^{2}} \delta_{i 3}
$$

where the diffusion term of the velocity potential disappears by the Laplace equation. After splitting the convection terms and rearranging, Equation (7) can be rewritten as follows:

$$
\left[\frac{\partial u_{p_{i}}}{\partial t}+u_{p_{j}} \frac{\partial u_{p_{i}}}{\partial x_{j}}-\frac{1}{F r^{2}} \delta_{i 3}\right]+\frac{\partial u_{i}^{*}}{\partial t}+u_{j}^{*} \frac{\partial u_{i}^{*}}{\partial x_{j}}+u_{j}^{*} \frac{\partial u_{p_{i}}}{\partial x_{j}}+u_{p_{j}} \frac{\partial u_{i}^{*}}{\partial x_{j}}=-\frac{\partial p}{\partial x_{i}}+\frac{1}{R e} \nabla^{2} u_{i}^{*}
$$

Using the Euler equation which can be obtained by setting the kinematic viscosity $v=0$ in the Navier-Stokes equations (Equation (5)), the bracket in Equation (8) becomes the potential pressure term, $\partial p_{\mathrm{p}} / \partial x_{i}$ (the subscript ' $\mathrm{p}$ ' means the potential pressure). This substitution eliminates the gravity force term from Equation (8), which consequently is written as

$$
\frac{\partial u_{i}^{*}}{\partial t}+u_{j}^{*} \frac{\partial u_{i}^{*}}{\partial x_{j}}+u_{j}^{*} \frac{\partial u_{p_{i}}}{\partial x_{j}}+u_{p_{j}} \frac{\partial u_{i}^{*}}{\partial x_{j}}=-\frac{\partial p^{*}}{\partial x_{i}}+\frac{1}{R e} \nabla^{2} u_{i}^{*}
$$

where the new pressure field, $p^{*}$, is defined as

$$
p^{*}=p-p_{\mathrm{p}}
$$

Equation (9) is of the same form as the original RANS equations but it includes the influence from the potential solution in the form of two new convection terms in the left hand side (boxed terms). For reference, we label the two additional terms as follows:

$$
\mathbf{A}=u_{j}^{*} \frac{\partial u_{p_{i}}}{\partial x_{j}} \quad \text { and } \quad \mathbf{B}=u_{p_{j}} \frac{\partial u_{i}^{*}}{\partial x_{j}}
$$

The first one, $u_{j}^{*} \partial u_{p_{i}} / \partial x_{j}$, corresponds to the convection of $u_{p_{i}}$ with the speed of $u_{i}^{*}$ and the second one, $u_{p_{j}} \partial u_{i}^{*} / \partial x_{j}$, corresponds to the convection of $u_{i}^{*}$ with the speed of $u_{p_{i}}$. Although we 
can choose any potential for the decomposition, it is desirable to have an appropriate potential for the inviscid field so that $u_{i}^{*}$ vanishes as quickly as possible. The set of Equations (4) and (9) constitute the complementary Navier-Stokes equations.

The complementary velocity $u_{i}^{*}$ can be decomposed into a mean part and a fluctuating part just as $u_{i}$, (i.e. $u_{i}^{*}=\overline{u_{i}^{*}}+u_{i}^{\prime}$ ). It should be noted that the fluctuating part is denoted as $u_{i}^{\prime}$, not $u_{i}^{* \prime}$. This means that the fluctuating part of $u_{i}^{*}$ is equal to that of $u_{i}$. This is clear if the decomposition is written as follows:

$$
u_{i}=\overline{u_{i}}+u_{i}^{\prime}=u_{p_{i}}+\overline{u_{i}^{*}}+u_{i}^{\prime}
$$

where the mean velocity, $\overline{u_{i}}$, becomes $u_{p_{i}}+\overline{u_{i}^{*}}$ and the fluctuating parts are the same in both decompositions.

The rest of the derivation of the complementary RANS equations is the same as that followed for the conventional RANS equations. That is, the complementary RANS equations can be obtained by substituting the decomposition shown in Equation (12) into the complementary Navier-Stokes equations and taking the time average in the conventional sense.

Using the eddy viscosity model the following form of the complementary RANS equations can be obtained:

$$
\frac{\partial u_{i}^{*}}{\partial t}+u_{j}^{*} \frac{\partial u_{i}^{*}}{\partial x_{j}}+u_{j}^{*} \frac{\partial u_{p_{i}}}{\partial x_{j}}+u_{p_{j}} \frac{\partial u_{i}^{*}}{\partial x_{j}}=-\frac{\partial P^{*}}{\partial x_{i}}+\frac{\partial}{\partial x_{j}}\left[\left(\frac{1}{R e}+v_{t}\right)\left(\frac{\partial u_{i}^{*}}{\partial x_{j}}+\frac{\partial u_{j}^{*}}{\partial x_{i}}\right)\right]
$$

where $v_{t}$ is the eddy viscosity, which is dependent on flow conditions and $P^{*}$ is defined as, $P^{*}=p^{*}+\frac{2}{3} k$ ( $k$ is the turbulent kinetic energy).

The conventional $k-\varepsilon$ turbulence closure of Chen and Patel [18] is chosen to model turbulence in this study. Following a similar procedure to that explained for the derivation of the complementary RANS equations, the corresponding modified $k-\varepsilon$ model is derived as

$$
\begin{aligned}
& \frac{\partial k}{\partial t}+u_{j}^{*} \frac{\partial k}{\partial x_{j}}+u_{p_{j}} \frac{\partial k}{\partial x_{j}}=\frac{\partial}{\partial x_{j}}\left[\left(\frac{1}{R e}+\frac{v_{t}}{\sigma_{k}}\right) \frac{\partial k}{\partial x_{j}}\right]+G-\varepsilon \\
& \frac{\partial \varepsilon}{\partial t}+u_{j}^{*} \frac{\partial \varepsilon}{\partial x_{j}}+u_{p_{j}} \frac{\partial \varepsilon}{\partial x_{j}}=\frac{\partial}{\partial x_{j}}\left[\left(\frac{1}{R e}+\frac{v_{t}}{\sigma_{\varepsilon}}\right) \frac{\partial \varepsilon}{\partial x_{j}}\right]+C_{\varepsilon_{1}} \frac{\varepsilon}{k} G-C_{\varepsilon_{2}} \frac{\varepsilon^{2}}{k}
\end{aligned}
$$

where $\varepsilon$ is the turbulent energy dissipation rate. The eddy viscosity is modelled as, $v_{t}=C_{\mu} k^{2} / \varepsilon$, and $G$ is the production term defined as

$$
G=-\overline{u_{i}^{\prime} u_{j}^{\prime}} \frac{\partial u_{i}}{\partial x_{j}}=v_{t}\left(\frac{\partial u_{i}}{\partial x_{j}}+\frac{\partial u_{j}}{\partial x_{i}}\right) \frac{\partial u_{i}}{\partial x_{j}}
$$

The diffusion rate of $k$ and $\varepsilon$ are $\sigma_{k}$ and $\sigma_{\varepsilon}$ and $C_{\varepsilon_{1}}, C_{\varepsilon_{2}}$ and $C_{\mu}$ are empirical constants. In this study wall functions are employed to avoid the integration of the equations through the sublayer. The conventional wall function formulation derived by Chen and Patel [19] is used herein. 


\section{NUMERICAL METHODOLOGY}

In this study, we consider a deeply submerged body moving in the negative $x$ direction at constant speed $U_{0}$. The potential flow around the body is considered to be steady, inviscid and irrotational. The flow velocity potential can then be expressed as

$$
\Phi=U_{0} x+\phi
$$

where $\phi$ is a perturbation potential.

A Rankine source type desingularized method of Beck [20] is employed for the potential solver with sources located inside the body. Unlike the traditional panel method, the kernel function is not singular when desingularized sources are used. The potential can be written at any point of the domain, say $i$, as

$$
\phi_{i}=\sum_{j=1}^{N} \sigma_{j} G_{i j}
$$

where $N$ is the number of all sources in the domain that influence the potential at any point $i, \sigma_{j}$ is the strength of the $j$ th source and $G_{i j}$ is the influence function. For two-dimensional problems in this study, a logarithmic function $\left(G_{i j}\left(\mathbf{x}_{\mathrm{c} i}, \mathbf{x}_{\mathrm{s} j}\right)=1 / 2 \pi \ln r_{i j}\right)$ is used, where $\mathbf{x}_{\mathrm{c} i}$ and $\mathbf{x}_{\mathrm{s} i}$ are the position vectors corresponding to any field point and source point respectively, and $r_{i j}$ is the distance between both field point and source points.

The boundary conditions used are a radiation condition on the outer boundary to ensure the recovery of the free stream velocity $U_{0}$, and a no-flow-through condition at the body, that is

$$
\frac{\partial \phi}{\partial n}=-U_{0} n_{1}
$$

where $n_{1}$ is the $x$-component of the normal vector into the body.

A matrix equation is then constructed by combining Equations (17) and (18) and the LU decomposition method is used to solve for the source strengths, $\sigma_{i}$. Once these source strengths are identified, any velocity, and consequently any pressure can be determined at any field point, $\mathbf{x}_{\mathrm{c}}$, by summing the influences from all the sources. The general formulation of the potential velocity is as follows:

$$
u_{p_{i}}=U_{0}+\sum_{j=1}^{N} \frac{1}{2 \pi} \sigma_{j} \frac{x_{c i}-x_{s j}}{r_{i j}^{2}}, \quad v_{p_{i}}=\sum_{j=1}^{N} \frac{1}{2 \pi} \sigma_{j} \frac{y_{c_{i}}-y_{s_{j}}}{r_{i j}^{2}}
$$

where $u_{p_{i}}$ and $v_{p_{i}}$ are the components of the potential velocity at any field point $i,\left(x_{c i}, y_{c_{i}}\right)$ are the co-ordinates of the position vector $\mathbf{x}_{\mathrm{c}}$ at the field point $i$, and $\left(x_{s j}, y_{s_{j}}\right)$ are the co-ordinates of the position vector $\mathbf{x}_{\mathrm{s}}$ at a source point $j$.

An existing RANS solver, used in many different simulations of internal and external turbulent flows [21,22], was used as the starting point to test the complementary RANS equations in generalized curvilinear co-ordinates $\left(\xi^{1}, \xi^{2}, \xi^{3}\right)$. The corresponding continuity and complementary RANS equations in generalized curvilinear co-ordinates can be written as follows:

$$
J \frac{\partial}{\partial \xi^{i}}\left(\frac{V^{* i}}{J}\right)=0
$$




$$
\begin{aligned}
& \frac{\partial u_{i}^{*}}{\partial t}+V^{* j} \frac{\partial u_{i}^{*}}{\partial \xi^{j}}+V_{p}^{j} \frac{\partial u_{i}^{*}}{\partial \xi^{j}}+V^{* j} \frac{\partial u_{p_{i}}}{\partial \xi^{j}} \\
& =-\frac{\partial P^{*}}{\partial \xi^{j}} \xi_{x_{i}}^{j}+J \frac{\partial}{\partial \xi^{j}}\left(\frac{1}{J}\left\{v_{T}\left[g^{p j} \frac{\partial u_{i}^{*}}{\partial \xi^{p}}+\xi_{x_{p}}^{j} R_{p i}\right]\right\}\right)
\end{aligned}
$$

where the last two terms on the left hand side of the momentum equation represent the two additional terms due to the inclusion of the potential solution. To derive Equations (20) and (21) a partial transformation is used leaving the velocity, $u_{i}^{*}$, in Cartesian co-ordinates for simplicity, while the rest of the vectors are expressed in generalized curvilinear co-ordinates. In Equations (21), $J, \xi_{x_{k}}^{j}$, and $g^{i j}$ represent the Jacobian, the metrics, and the contravariant metric tensor of the geometric transformation, respectively, and they are defined as

$$
\begin{aligned}
J=\frac{\partial\left(\xi^{1}, \xi^{2}, \xi^{3}\right)}{\partial\left(x_{1}, x_{2}, x_{3}\right)} & =\operatorname{det}\left|\begin{array}{lll}
\xi_{x_{1}}^{1} & \xi_{x_{2}}^{1} & \xi_{x_{3}}^{1} \\
\xi_{x_{1}}^{2} & \xi_{x_{2}}^{2} & \xi_{x_{3}}^{2} \\
\xi_{x_{1}}^{3} & \xi_{x_{2}}^{3} & \xi_{x_{3}}^{3}
\end{array}\right| \\
\xi_{x_{k}}^{j} & =\frac{\partial \xi^{j}}{\partial x_{k}} \\
g^{i j} & =\xi_{x_{k}}^{i} \xi_{x_{k}}^{j}
\end{aligned}
$$

and $V^{* j}$ and $V_{p}^{j}$ are the contravariant velocity components corresponding to $V^{*}$ and $V_{p}$, respectively, defined as

$$
V^{* j}=u_{k}^{*} \xi_{x_{k}}^{j}, \quad V_{p}^{j}=u_{p_{k}} \xi_{x_{k}}^{j}
$$

In the same Equation (21), the tensor $R_{i j}$ is defined as

$$
R_{i j}=\frac{\partial u_{i}^{*}}{\partial \xi^{k}} \xi_{x_{j}}^{k}
$$

Similarly the corresponding modified $k-\varepsilon$ turbulence model in generalized curvilinear co-ordinates is developed and results in

$$
\begin{aligned}
& \frac{\partial k}{\partial t}+V^{* j} \frac{\partial k}{\partial \xi^{j}}+V_{p}^{* j} \frac{\partial k}{\partial \xi^{j}}=J \frac{\partial}{\partial \xi^{j}}\left[\left(\frac{1}{R e}+\frac{v_{t}}{\sigma_{k}}\right) \frac{g^{p j}}{J} \frac{\partial k}{\partial \xi^{p}}\right]-G+\varepsilon \\
& \frac{\partial \varepsilon}{\partial t}+V^{* j} \frac{\partial \varepsilon}{\partial \xi^{j}}+V_{p}^{* j} \frac{\partial \varepsilon}{\partial \xi^{j}}=J \frac{\partial}{\partial \xi^{j}}\left[\left(\frac{1}{R e} \frac{v_{t}}{\sigma_{\varepsilon}}\right) \frac{g^{p j}}{J} \frac{\partial \varepsilon}{\partial \xi^{p}}\right]-C_{\varepsilon_{1}} \frac{\varepsilon}{k} G+C_{\varepsilon_{2}} \frac{\varepsilon^{2}}{k}
\end{aligned}
$$

The continuity and momentum equations are discretized on a non-staggered mesh. The continuity equation is discretized in space using three-point central finite differencing. The same discretization algorithm is used in the momentum equations for the pressure gradient and the diffusion terms. Both first- and second-order accurate flux splitting based upwind differencing schemes [23] are tried in this study for the convection terms. As noted earlier in Section 2 the two extra terms that appear in the complementary RANS equations are convection terms. The upwind differencing of the convection terms eliminates the need for 
explicitly adding artificial dissipation terms to the right hand side of the momentum equations to stabilize the numerical algorithm.

In converting a conventional RANS code to a complementary RANS code, consistency must be kept in the numerical scheme and special care must be taken in the numerical implementation of the additional terms, as a problem may arise with the second additional term of Equation (13). Define $\mathbf{P}$ and $\mathbf{Q}$ as follows:

$$
\begin{aligned}
& \mathbf{P} \equiv\left(\mathbf{u}^{*} \cdot \nabla\right) \mathbf{u}^{*}+(\nabla \Phi \cdot \nabla) \mathbf{u}^{*} \\
& \left.\mathbf{Q} \equiv\left[\left(\mathbf{u}^{*}+\nabla \Phi\right) \cdot \nabla\right)\right] \mathbf{u}^{*}
\end{aligned}
$$

where $\mathbf{P}$ is the summation of the convection term and the second additional term in Equation (13), and $\mathbf{Q}$ is the combination of the two terms in $\mathbf{P}$.

In the upwind scheme used in this study, the direction of the differencing for the gradient is determined by the sign of the velocity multiplying the gradient, i.e. $\mathbf{u}^{*}$ and $\nabla \Phi$ for $\mathbf{P}$ and $\left(\mathbf{u}^{*}+\nabla \Phi\right)$ for $\mathbf{Q}$. For instance, if $\nabla \Phi$ is positive and greater than the total velocity $\mathbf{u}$, which is also positive, then $\mathbf{u}^{*}$ becomes negative (recall Equation (3)). Therefore, the gradients in $\mathbf{P}$ are discretized by a backward and a forward differencing for the first and the second terms, respectively, while the gradient in $\mathbf{Q}$ is discretized by a forward differencing. These differences in the directions of the discretization could cause large discrepancies between $\mathbf{P}$ and $\mathbf{Q}$. This is especially true where the velocity gradients are large, such as the leading edge or the trailing edge of an airfoil. The combined term, $\mathbf{Q}$, on the other hand, uses the total velocity $\left(\left(\mathbf{u}^{*}+\nabla \Phi\right)\right.$ or $\left.\mathbf{u}\right)$ to determine the direction of the differencing. In some of our computations, the use of $\mathbf{P}$ instead of $\mathbf{Q}$ caused up to $4 \%$ RMS difference between the conventional RANS equations and the complementary RANS equations. In this study the second additional term is combined with the convection term of the classical RANS equations for all the simulations presented.

An implicit fractional-step method of Chorin [24] is used to integrate the RANS equations in time to steady state, that is until no further change in the solutions is observed. The $x$-equation corresponding to the complementary RANS equations can be written as follows:

$$
\frac{\partial u^{*}}{\partial t}+\mathbf{C}\left(u^{* n}+\Delta u^{*}\right)+\mathbf{A}\left(u_{p}\right)+\mathbf{H}\left(P^{* n}+\Delta P\right)-\mathbf{D}\left(u^{* n}+\Delta u^{*}\right)=0
$$

where $\mathbf{C}, \mathbf{A}, \mathbf{H}$, and $\mathbf{D}$ represent the convection term (note that this convection term, $\mathbf{C}$, results from the combination of the convection term in the traditional RANS equations and the second additional convection term in Equation (21)), the first additional term in Equation (21), the pressure term, and the diffusion term, respectively. The superscript ' $n$ ' indicates the current time step, while ' $n+1$ ' refers to the next time step. The intermediate time step defined for the velocity field, as mentioned earlier, is denoted as ' $m$ ', and the streamwise component of the velocity at the intermediate time step is $\left(u^{* m}=u^{* n}+\Delta u^{*}\right)$.

A similar derivation to Equation (29) applies for the complementary RANS equations in the other two directions $y$ and $z$ (not shown here). Equation (29) can be split into the following two equations by employing an explicit method for the time derivative term and an implicit method for the other terms:

$$
\Delta u^{*}+\Delta t\left[\mathbf{C}\left(\Delta u^{*}\right)-\mathbf{D}\left(\Delta u^{*}\right)\right]=-\Delta t \cdot \mathrm{RHS}
$$


and

$$
\Delta t \cdot \mathbf{H}\left(\Delta P^{*}\right)=-\left(u^{* n+1}-u^{* m}\right)
$$

where

$$
\mathrm{RHS} \equiv \mathbf{C}\left(u^{* n}\right)+\mathbf{A}\left(u_{p}^{n}\right)-\mathbf{D}\left(u^{* n}\right)+\mathbf{H}\left(P^{* n}\right)
$$

Both equations are solved by using the approximate factorization method of Beam and Warming [25]. In the approximate factorization method, the original multi-dimensional difference equations are replaced by a series of one-dimensional difference equations, which can then be formulated by a tri-diagonal matrix equation. The method allows an efficient solution of relatively complex systems of differential equations [26]. A fourth-order artificial source term used by Sotiropoulos and Abdallah [27] is added to the right hand side of the continuity equation to avoid odd-even decoupling of the pressure field related to the use of a non-staggered grid.

A similar numerical procedure to that used to discretize and solve the momentum equations is applied for the equations defining the turbulence closure.

\section{DISCUSSION OF RESULTS}

To assess the performance of the numerical solver, three flows were simulated by solving the complementary RANS equations. The results were compared to analytical and experimental results where pertinent, as well as to the simulations resulting from solving the traditional RANS equations. The purpose of undertaking this initial validation study was two fold. First to verify that the complementary RANS equations give the same results as the conventional RANS equations within a desired accuracy and secondly to check on the benefits of the new proposed approach to solve viscous flows.

The three flows chosen to validate the numerical results of the complementary equations are the laminar and turbulent flows in a square duct, over a flat plate and over a NACA airfoil. The results corresponding to the flow in a square duct are not included here due to the simplicity of the potential flow (uniform flow) employed (refer to Kim et al. [28]). The results show excellent agreement with the analytic solution in Reference [29] and the experimental data of Humphrey et al. [30].

Hereafter, ' $u$-code' represents the solutions computed by the conventional RANS equations, and ' $\mathrm{u}$ *-code' represents the solutions from the complementary RANS equations. In all numerical computations presented herein the code was run until the residuals, defined as the summation of differences between the current and the previous iterations, were reduced by at least four orders of magnitude. Most computations were run on an SGI Origin 3400 computer system.

\subsection{Flow over a flat plate}

The steady laminar and turbulent flows over a two-dimensional flat plate were computed and the results are presented next. A discussion on the effects due to the choice of the potential solution is also included in this section. 


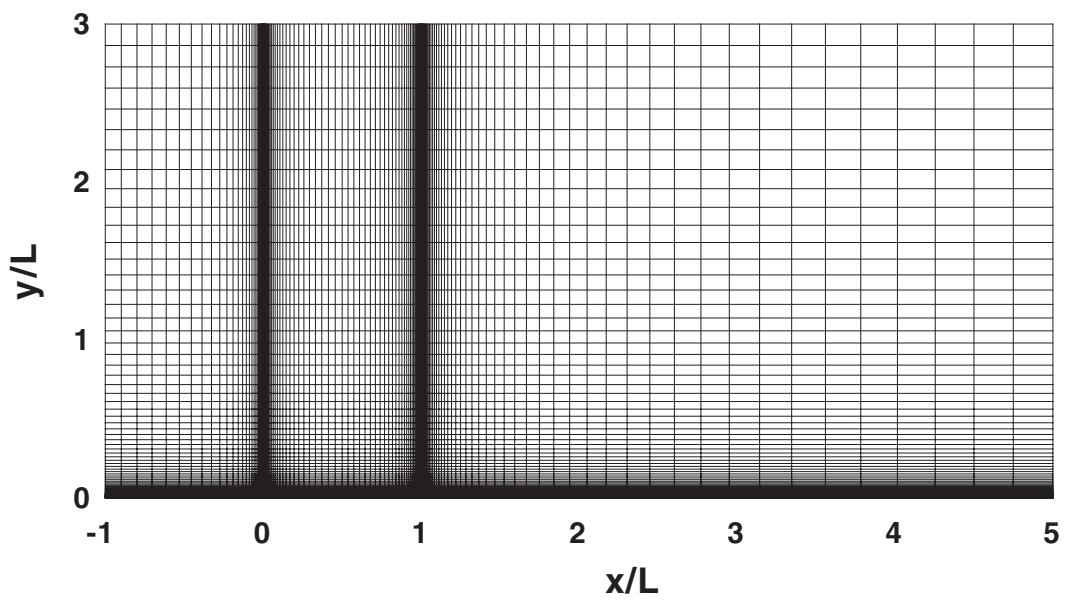

Figure 2. Computational domain and grid (flat plate).

4.1.1. Laminar flow over the flat plate. The laminar flow computations were performed at $R e=7900$, where $R e$ is based on the plate length. Figure 2 shows the computational grid, in which the plate is located on the $x$-axis between 0 and 1 . The number of nodes used is $141 \times 81$, and the grid is clustered in the boundary layer and also near the leading edge and the trailing edge using a hyperbolic tangent stretching function [31].

The boundary conditions for the 'u-code' are a free stream condition applied at the inlet and the top boundary, a symmetry boundary condition applied to the centreline, upstream and downstream of the plate, a no-slip condition applied on the plate, and a Neumann condition applied at the exit. The boundary conditions imposed on $u_{i}^{*}$ in the ' $\mathrm{u}$ *-code' are obtained by substituting the velocity decomposition equation (Equation (3)) into the boundary conditions of $u_{i}$ used for the conventional RANS equations. Consequently the wall, inlet and exit boundary conditions for $u_{i}^{*}$ can become

$$
\begin{aligned}
u_{i}^{*} & =-\left.u_{p_{i}}\right|_{\text {on wall }} \\
u_{i}^{*} & =U_{0} \hat{i}-\left.u_{p_{i}}\right|_{\text {at inlet }} \\
\frac{\partial u_{i}^{*}}{\partial x} & =-\left.\frac{\partial u_{p_{i}}}{\partial x}\right|_{\text {at outlet }}
\end{aligned}
$$

For the symmetry plane, the normal velocity to the plane of symmetry is set to zero and the gradient of the other velocity components is forced to vanish. Recovery of the free stream is enforced at the outer boundary. It should be noted that due to the choice of the outer boundary condition, the edge velocity, $U_{\mathrm{e}}$, of the boundary layer was chosen as the velocity scale, where the edge velocity is defined as the maximum horizontal velocity at any given $x$-location. 

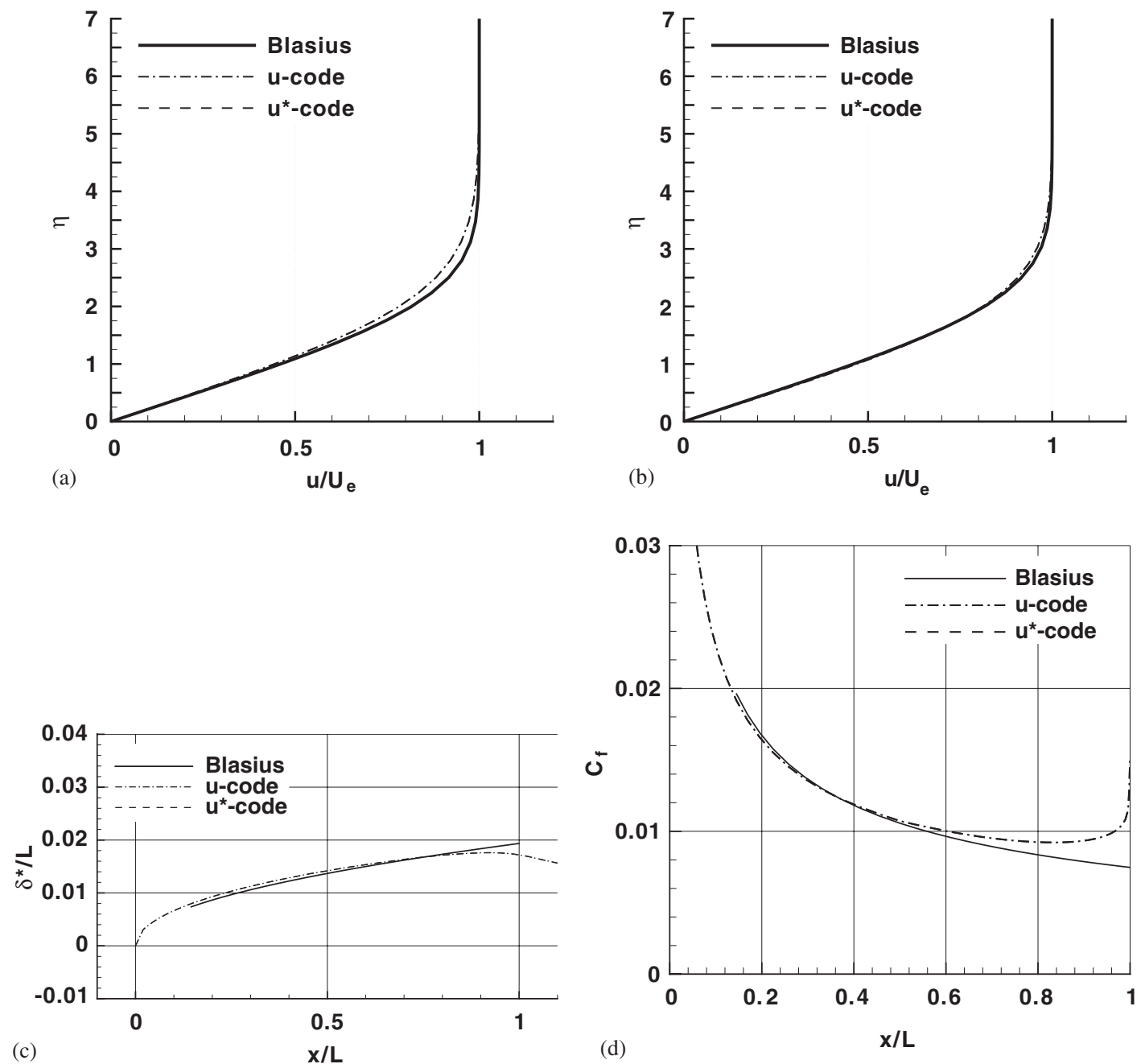

Figure 3. Velocity profiles, displacement thickness, and friction coefficient distributions (flat plate, $R e=7900$ ): (a) at $x / L=0.2$; (b) at $x / L=0.7$; (c) displacement thickness; and (d) friction coefficient.

The computational results were compared to the Blasius solution [32] and the u-code results. Figure 3 shows the comparisons of streamwise velocity components, displacement thickness, and friction coefficient. Figures 3(a) and (b) show the streamwise velocity components versus the non-dimensional parameter $\eta$, defined as $\eta=y \sqrt{U_{0} / 2 v x}$. Small discrepancies are observed between the Blasius solution and the two numerical solutions in Figure 3(a), due to the proximity of the $x / L$ location to the leading edge, where the Blasius solution does not apply. In Figure $3(\mathrm{~b})$, the $\mathrm{u}^{*}$-code shows good agreement with both the Blasius solution and the u-code solution. 
To assess the differences between solutions, a root-mean-square (RMS) difference was calculated. The RMS difference is defined as

$$
\text { RMS error }(\%)=\frac{\sqrt{\sum\left(u_{1}-u_{2}\right)^{2} / N}}{U_{0}} 100
$$

where $u_{1}$ and $u_{2}$ are the solution to be compared, $N$ is the number of nodes. The RMS differences of the $u^{*}$-code solution with respect to the Blasius solution are about $1 \%$ of the free stream. The RMS differences between the $\mathrm{u}$-code and the $\mathrm{u}^{*}$-code are less than $0.0018 \%$ of the free stream.

Figure 3(c) shows the comparison of the evolution of the displacement thickness, $\delta^{*}$, (i.e. $\left.\delta^{*}=\int_{0}^{\delta_{99 \%}}\left(1-u / U_{\mathrm{e}}\right) \mathrm{d} y\right)$ in the $x$-direction between the Blasius solution, the u-code solution, and the $\mathrm{u}^{*}$-code solution. As the figure shows, the $\mathrm{u}^{*}$-code and the $\mathrm{u}$-code show excellent agreement. There are some, to be expected, discrepancies between the Blasius solution and the $\mathrm{u}^{*}$-code solution near the leading edge and the trailing edge due to the limitations of the analytical derivation. Figure $3(\mathrm{~d})$ shows the friction coefficients, $C_{\mathrm{f}}$, (i.e. $C_{\mathrm{f}}(x)=\tau_{\mathrm{w}}(x) / \frac{1}{2} \rho U_{0}^{2}$, where $\tau_{\mathrm{w}}$ is the wall shear stress) from the Blasius solution, the u-code solution and the $\mathrm{u}^{*}$-code solution. As expected the agreement between the $\mathrm{u}$ - and $\mathrm{u}^{*}$-codes is excellent along the plate and the agreement with the Blasius solution is good for $0.1<x / L<0.8$. Disagreement at both the leading edge and the trailing edge is again to be expected.

4.1.2. Effect of the choice of the potential function. In order to verify that regardless of the potential flow used the complementary RANS equations give consistent solutions, several tests were performed. Three potentials were tested; a uniform potential, a potential with a corresponding linear velocity distribution, denoted as 'linear $\Phi$ ' and a potential with sinusoidal sources and vortices, denoted as 'non-uniform $\Phi$ '.

The linear $\Phi$ is given by

$$
\Phi=U_{0} x+A_{0}\left[\left(x+x_{0}\right)^{2}-y^{2}\right]
$$

where $A_{0}$ is a constant, which determines the magnitude of the perturbation velocities and $x_{0}$ is a constant, which shifts the origin of the profiles to the inlet such that the symmetry of the potential solution is satisfied. The non-uniform $\Phi$ is given by

$$
\Phi=U_{0} x+\sum_{j}^{N} \frac{1}{2 \pi}\left[\sigma_{j} \log r_{i j}+\gamma_{j} \theta_{i j}\right]
$$

where $\sigma$ is the source strength, and $\gamma$ is the vortex strength, $N$ is the number of sources or vortices, $r_{i j}$ is the distance between a field point and a source point, and $\theta_{i j}$ is the angle between the field point and the source point. The source strength, $\sigma$ and the vortex strength, $\gamma$ are given by

$$
\begin{gathered}
\sigma(x)=\sigma_{0} \cos \left[a_{0}\left(x+x_{0}\right)\right] \\
\gamma(x)=\gamma_{0} \sin \left[a_{0}\left(x+x_{0}\right)\right]
\end{gathered}
$$

where $\sigma_{0}, \gamma_{0}$ determine the amplitudes and $a_{0}$ determines the frequency of the profiles of the source and the vortex strengths. In the tests, $a_{0}$ was determined such that there are three cycles along $x$. As before, $x_{0}$ shifts the origin of sinusoidal curves to the inlet. 

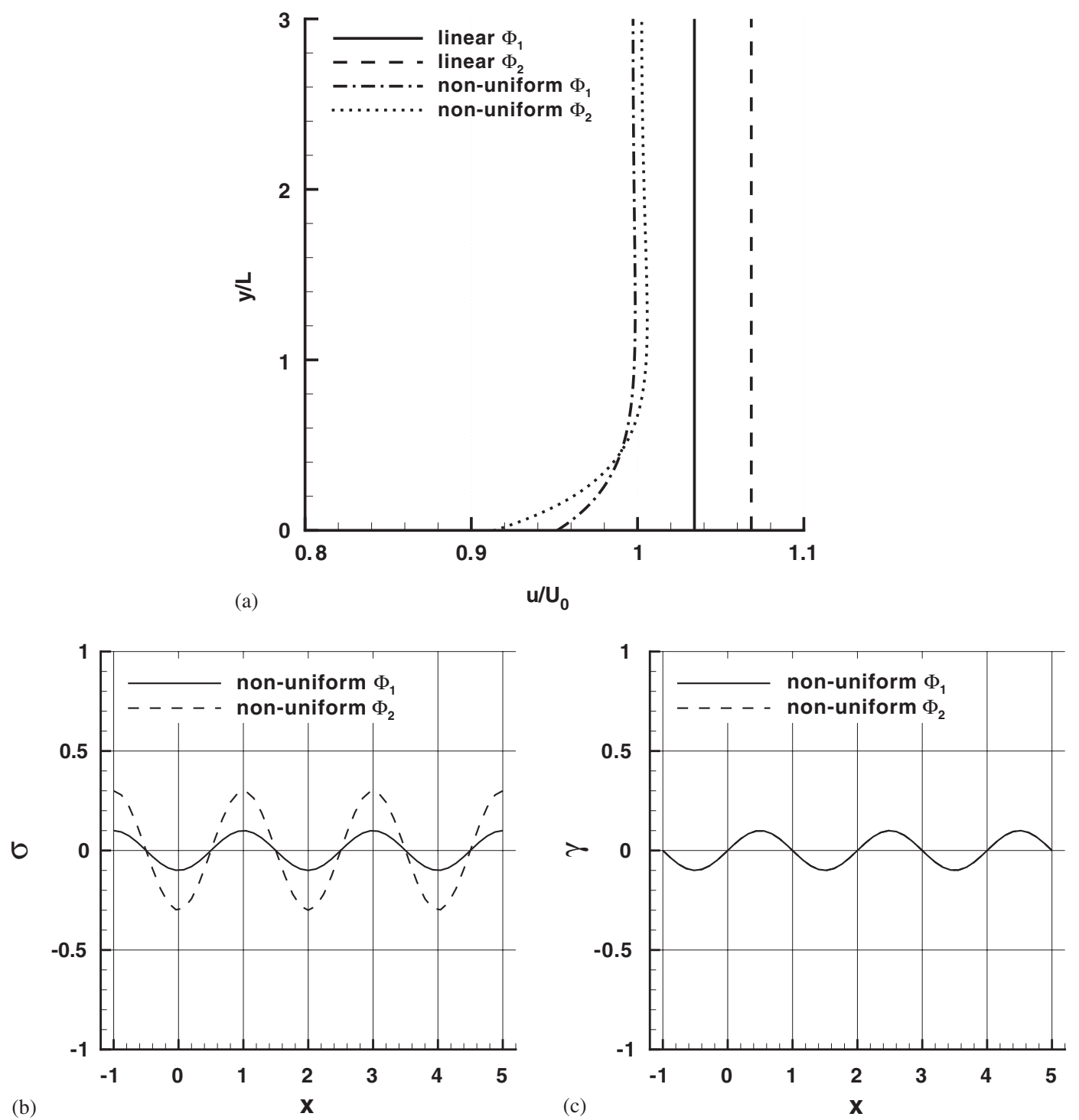

Figure 4. Potential functions (flat plate, $R e=7900$ ): (a) various potentials at $x / L=0.7$;

(b) source strength; and (c) vortex strength.

Figure 4(a) shows the corresponding horizontal velocity profiles for the linear and the nonuniform potentials at $x / L=0.7$. The linear potentials yield a constant $u_{p}$ in $y$ because they vary linearly in $x$. The non-uniform potentials show large gradients near the wall due to the strong sources and vortices close to the centreline. The source strength, $\sigma$ and the vortex strength $\gamma$ are shown in Figures 4(b) and (c). As shown, both $\sigma$ and $\gamma$ vary in a sinusoidal fashion and have three wavelengths across the domain. The amplitude of the vortex strength 
is fixed, while that of the source strength has two different values. The parameters used in the computations are $A_{0}=0.01$ for the linear $\Phi_{1}$ and $A_{0}=0.02$ for the linear $\Phi_{2}$, while for the non-uniform potentials $\Phi_{1}$ and $\Phi_{2} \sigma_{0}=0.1$ and 0.03 , respectively, were used with the same $\gamma_{0}=0.1$. These values lead to perturbation velocities that are on the order of $10 \%$ of the free stream. Fifty one source/vortex pairs were used and evenly spaced from the inlet to the exit along the horizontal line at $y / L=-0.8$ when the non-uniform $\Phi$ is used.

The RMS differences of the horizontal velocity were computed between the $\mathrm{u}^{*}$-code with the uniform $\Phi$ and the other cases. The differences become slightly larger as the potentials become more complex, however, the RMS differences are all less than $0.6 \%$ of the free stream. The computed RMS values corresponding to the linear $\Phi_{1}$ and $\Phi_{2}$, and the non-uniform $\Phi_{1}$ and $\Phi_{2}$ are $0.11,0.21,0.28$, and $0.55 \%$ of the free stream, respectively.

4.1.3. Turbulent flow over the flat plate. The complementary RANS solver is extended to the computation of turbulent flows with $k-\varepsilon$ turbulence closure. The purpose of this flat plate computation is neither to develop a new turbulence model for the complementary RANS solver nor to predict more accurate turbulent characteristics. The purpose is to examine the viability of the complementary RANS solver in turbulent flow computations and to identify outstanding differences, if any, between the complementary and the conventional RANS solvers.

The computational domain is the same as that of the laminar flow computation. The Reynolds number is set to $1.6 \times 10^{6}$. The experimental results of DeGraaff and Eaton [33] and Ramaprian et al. [34], the numerical results of Kim et al. [35], and results from the commercial software, Fluent ${ }^{\circledR}$ are used for comparison with the present numerical results. As in the laminar flow computation, the first-order upwind scheme is used for the convection terms.

Free stream conditions are applied at the inlet and outer boundaries. On the plate all velocity components and turbulence quantities are set to zero. A mirror-image reflection is applied at the plane of symmetry, that is, Neumann conditions are applied for the horizontal velocity and turbulence quantities and the vertical velocity is set to zero. At the exit boundary the velocity components and turbulence quantities are linearly extrapolated from the interior nodes. Linear extrapolation is also used to compute the pressure at all boundaries. The grid $141 \times 81$ had the first node off the plate located at an average $y^{+}\left(=U_{\tau} y / v\right.$, where $\left.U_{\tau}=\sqrt{\tau_{\mathrm{w}} / \rho}\right)$ of 40 , which is within the valid range of the law of the wall, $30<y^{+}<350$ (refer to Reference $[29$, p. 415]).

Figure 5 shows the mean streamwise velocity, $u^{+}\left(=u / U_{\tau}\right)$ profile (Figure $5(\mathrm{a})$ ) and the normalized turbulence kinetic energy, $k^{+}$, profile (Figure $5(\mathrm{~b})$ ) at $x / L=0.33$. The location $x / L=0.33$ corresponds to $\operatorname{Re}_{\theta} \approx 1400$, where $\operatorname{Re}_{\theta}\left(=U_{0} \theta / v\right)$ is the Reynolds number based on the momentum thickness, $\theta$. Figures $5(\mathrm{a})$ and (b) show comparisons of the results from the complementary RANS equations with other experimental and numerical results. Good agreement is shown overall except for the region closest to the wall. This is to be expected, however, due to the use of wall functions which essentially avoids the computation of the flow in the near wall region.

Figures 6(a) and (b) show comparisons of the friction coefficient and the evolution of the centreline velocity predicted by the $\mathrm{u}^{*}$-code with other numerical and experimental results including the empirical fit of the friction coefficient by Coles [36].

Overall the RMS differences in the horizontal velocity between the u-code and the $\mathrm{u}^{*}$-code are only $0.0009 \%$ of the free stream. The RMS differences in $v$ and $P$ are 0.0004 and $0.0017 \%$ of the free stream, respectively. 


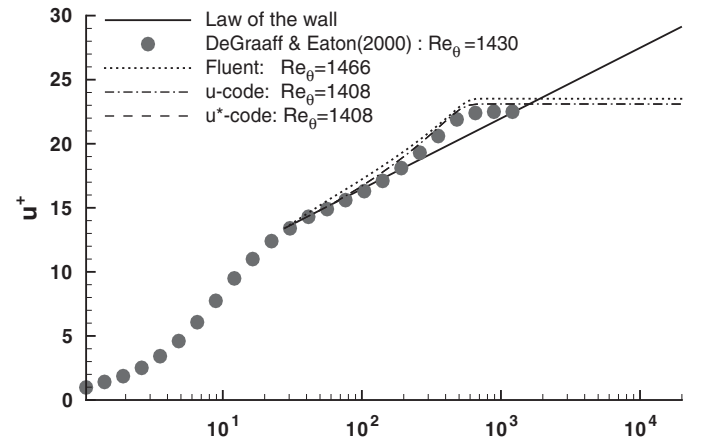

(a)

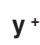

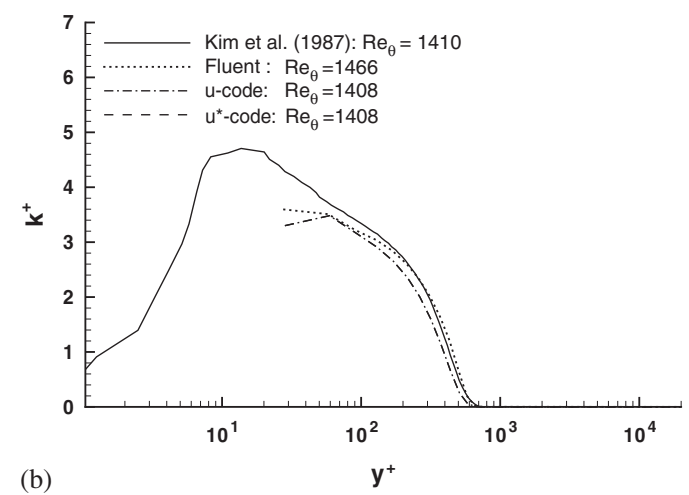

Figure 5. Mean streamwise velocity, $u^{+}$, and turbulent kinetic energy, $k^{+}$profiles (flat plate,

$$
\operatorname{Re}=1.6 \times 10^{6} \text { ): (a) } u^{+} \text {; and (b) } k^{+} \text {. }
$$
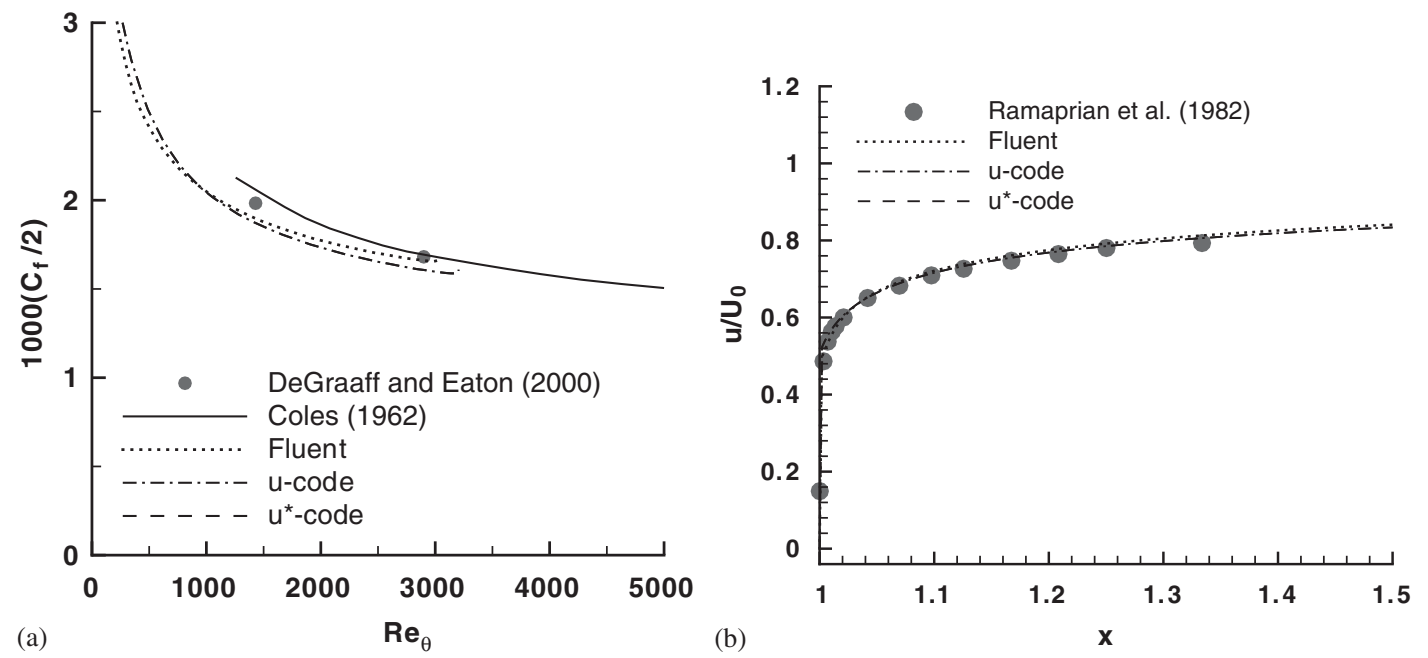

Figure 6. Friction coefficient and centreline velocity (flat plate, $R e=1.6 \times 10^{6}$ ): (a) friction coefficient; $C_{\mathrm{f}}$; and (b) centreline velocity.

\subsection{Flow over NACA airfoil}

4.2.1. Laminar flow over NACA 0010 foil. Computations for the steady laminar flow over a two-dimensional NACA 0010 airfoil at $R e=7900$ for which several different potential flows were used are presented next. The use of a uniform potential results in a $\mathrm{u}^{*}$-code solution that is identical to the u-code solution but shifted from one-another by the uniform stream value. Hence the $u^{*}$ solution does not decay any faster than the $u$ solution. Two body-fitted potentials were also employed: The first has singularities and their images inside the foil as shown in Figure 7 and the second potential has singularities distributed below the centreline in 


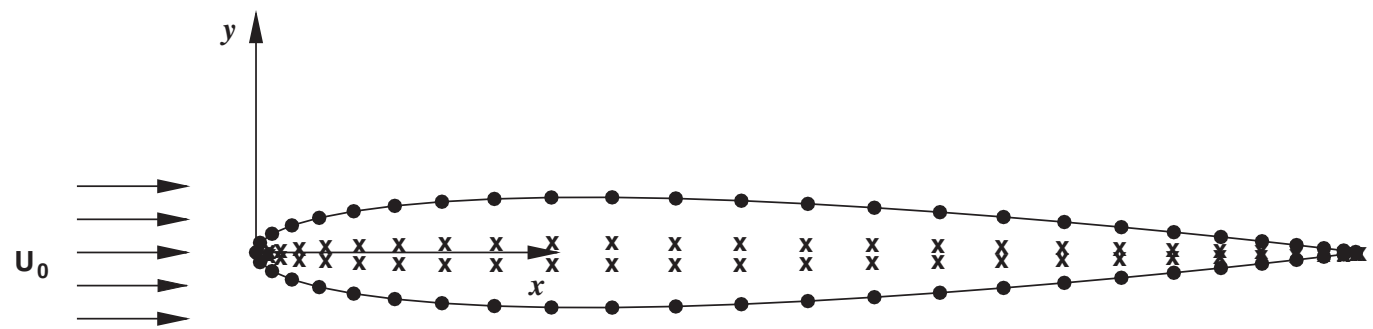

Figure 7. Co-ordinate system, control points $(\bullet)$ and source points $(\times)($ NACA 0010).
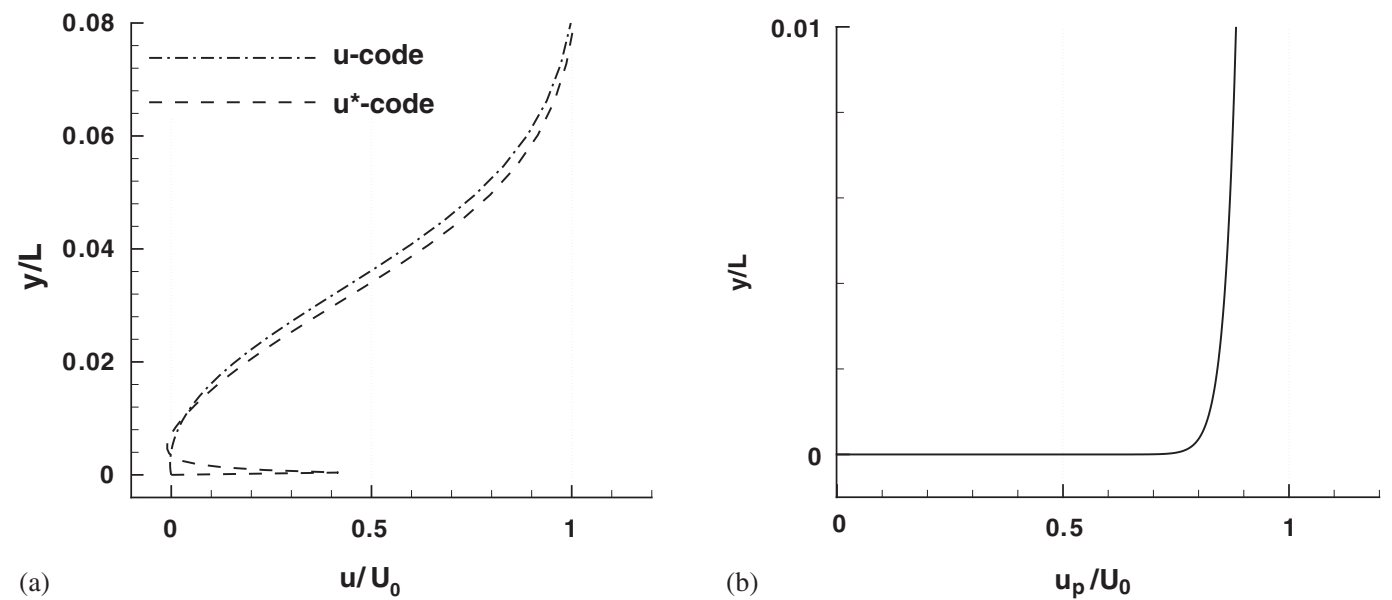

Figure 8. Horizontal velocities at the trailing edge (NACA 0010, $R e=7900$ ):

(a) $u$ and $u^{*}+u_{p}$; and (b) $u_{p}$.

the lower half plane as discussed later in this section. In the second potential, control points are distributed on the centreline upstream and downstream to ensure symmetric solutions. A Cartesian inertial co-ordinate system is located at the leading edge of the airfoil with the $y$-axis vertically upward and the $x$-axis in the direction of the flow. Figure 7 shows the co-ordinate system used for the computations.

The desingularized method of Beck [20] is used for the body-fitted potential solver. The sources are placed on the line of the normal vector from the control points as shown in Figure 7. The figure shows 51 sources and control points, however, a total of 359 sources were used in the computations. For the desingularized distance, the recommendation of Cao et al. [37] was followed while keeping the sources above $y=0$.

The results with the body-fitted potential show that while the $u^{*}$ solution should decay faster than the $u$ solution, that is not accomplished at the trailing edge. Figure 8(a) shows the $u$ profiles at the trailing edge. As shown, a large spike exists between the first and the second nodes in the $\mathrm{u}^{*}$-code result. This large spike is attributed to the sudden increase in the potential solution at the trailing edge as shown by the $u_{p}$ profile at the trailing edge in Figure 8(b). 
(a)

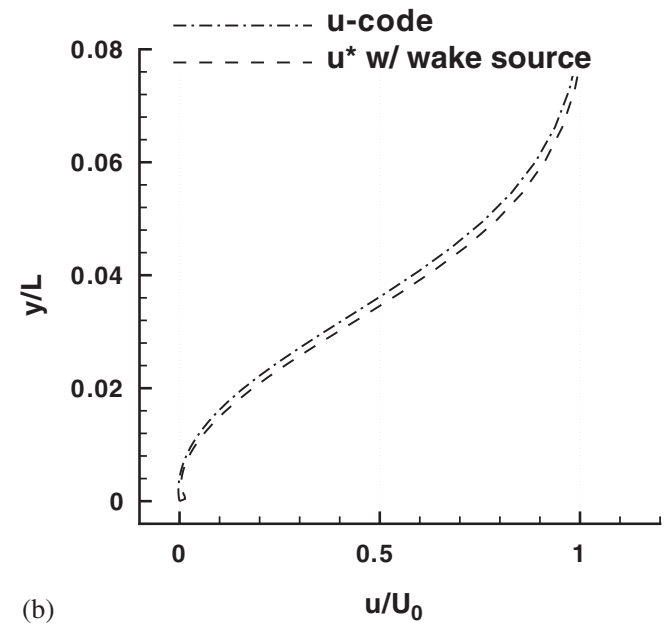

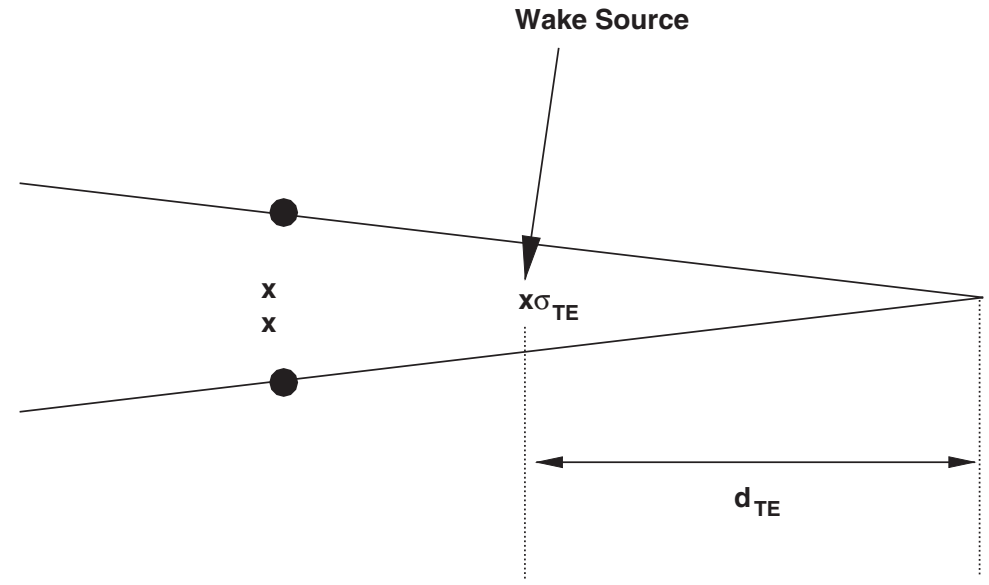

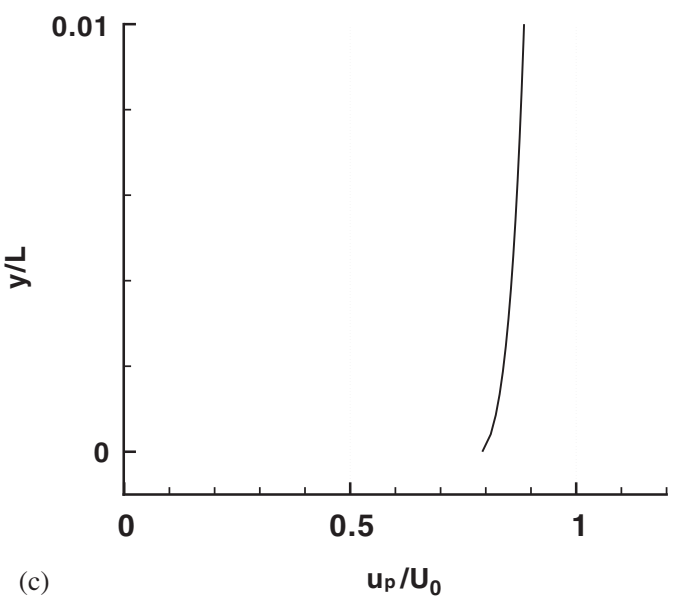

Figure 9. Schematic of the wake source and horizontal velocity profiles at the trailing edge (NACA 0010, $R e=7900$ ): (a) Wake source and parameters; (b) $u$ and $u^{*}+u_{p}$; and (c) $u_{p}$ with wake source.

An attempt to overcome this problem was made by extending the foil from a small distance above the centre line to $x=\infty$. Unfortunately, the potential flow could not be properly accounted for inside the extended body which made this option impractical since the potential solution must be known everywhere to solve the complementary RANS equations. Removal of the stagnation point at the trailing edge in the potential flow solution was also tried by replacing the control-source points at the trailing edge with a single source without a corresponding control point. The stagnation point can be avoided in the potential solution if this source strength is positive. There are two parameters to control this wake source; one is the source strength, $\sigma_{\mathrm{TE}}$, and the other is the distance from the trailing edge, $d_{\mathrm{TE}}$. Figure 9(a) shows a schematic of the wake source and parameters. As shown in Figure 9(c), the wake source had the desired effect of eliminating the stagnation point at the trailing edge. Hence, 


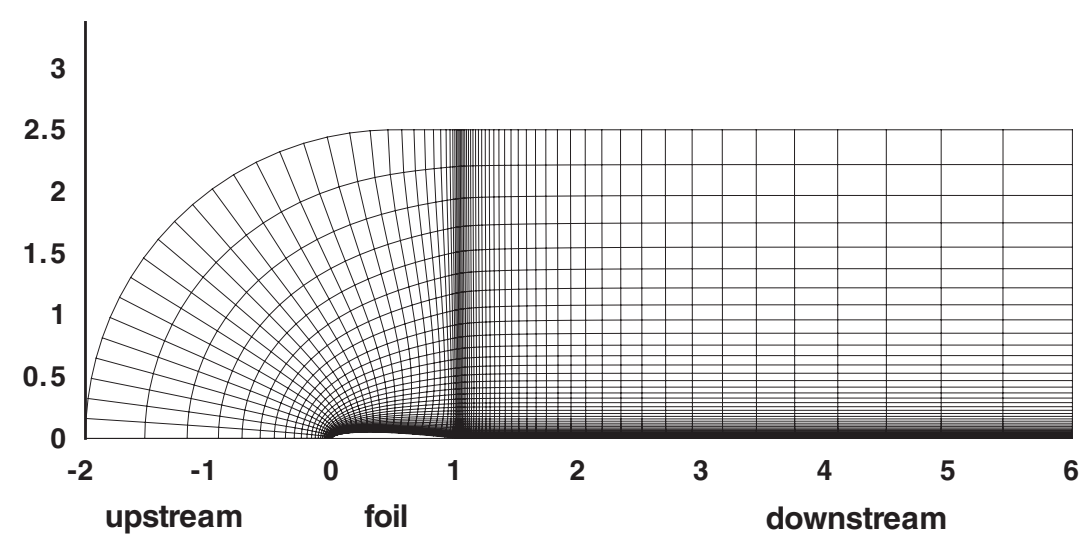

Figure 10. Computational domain and grid (NACA 0010).

the spike in the $u^{*}$-code disappears as shown in Figure 9(b). Consequently wake sources were used in all computations shown hereafter with $\sigma_{\mathrm{TE}}$ and $d_{\mathrm{TE}}$ chosen from a series of optimization tests, as $\sigma_{\mathrm{TE}}=0.1 \times 10^{-5}$ and $d_{\mathrm{TE}}=0.5 \times 10^{-4}$.

Figure 10 shows the computational domain and grid used for the simulations. The domain is two body lengths long upstream of the leading edge, five body lengths downstream of the trailing edge and two and a half body lengths in height. The grid shown in the figure has $71 \times 41$ grid points. The grid is generated by solving a Poisson differential equation of $\xi_{i}$ for $x_{i}$ following Thompson et al. [31]. The stretching ratio over almost the entire grid is close to 1.0. However, in the vicinity of the trailing edge, the maximum stretching ratio becomes as large as 2.0 and the minimum becomes 0.5 . The boundary conditions are similar to those employed in the flat plate problem (see Section 4.1). The numerical results presented in this section correspond to a CFL (Courant-Friedrichs-Lewy) number of 1.65 . The code was run with both first- and second-order upwind discretization schemes for the convection terms and the results will be compared and discussed later in this section.

A grid independence study is conducted for the $\mathrm{u}^{*}$-code and the u-code. Both are tested in a coarse $(71 \times 41)$, medium $(141 \times 81)$ and fine $(281 \times 161)$ grids. While both codes give consistent results, the $\mathrm{u}^{*}$-code gives less grid-dependent results than the $\mathrm{u}$-code.

Figures 11(a) and (b) show the horizontal velocity profiles at $x / L=0.54$ from the u-code and the $\mathrm{u}^{*}$-code, respectively. In the figure, the solutions corresponding to the coarse and the medium grids seem to converge to the results corresponding to the fine grid solutions. The $\mathrm{u}^{*}$-code solutions appear to be less grid dependent than the u-code solutions, since the $\mathrm{u}^{*}$-code solution at $x / L=0.54$ shows consistent results even in the coarse grid. More specifically the corner of the profile is well captured in the coarse grid as well as in the fine grid. On the other hand as shown in Figure 11(a), the u-code shows substantial difference amongst the various grids. This less grid dependency in the $\mathrm{u}^{*}$-code seems to be linked to the smaller gradients shown by the velocity profiles corresponding to the $\mathrm{u}^{*}$-code in comparison to those corresponding to the u-code. In other words, by introducing the potential solution, the velocity profiles computed with the $\mathrm{u}^{*}$-code may have less steep profiles than those computed with the u-code and consequently they may require less resolved grids for the same level of accuracy. Figure 11(c) shows the $u^{*}$ profiles at the same location for the various grids tested, 

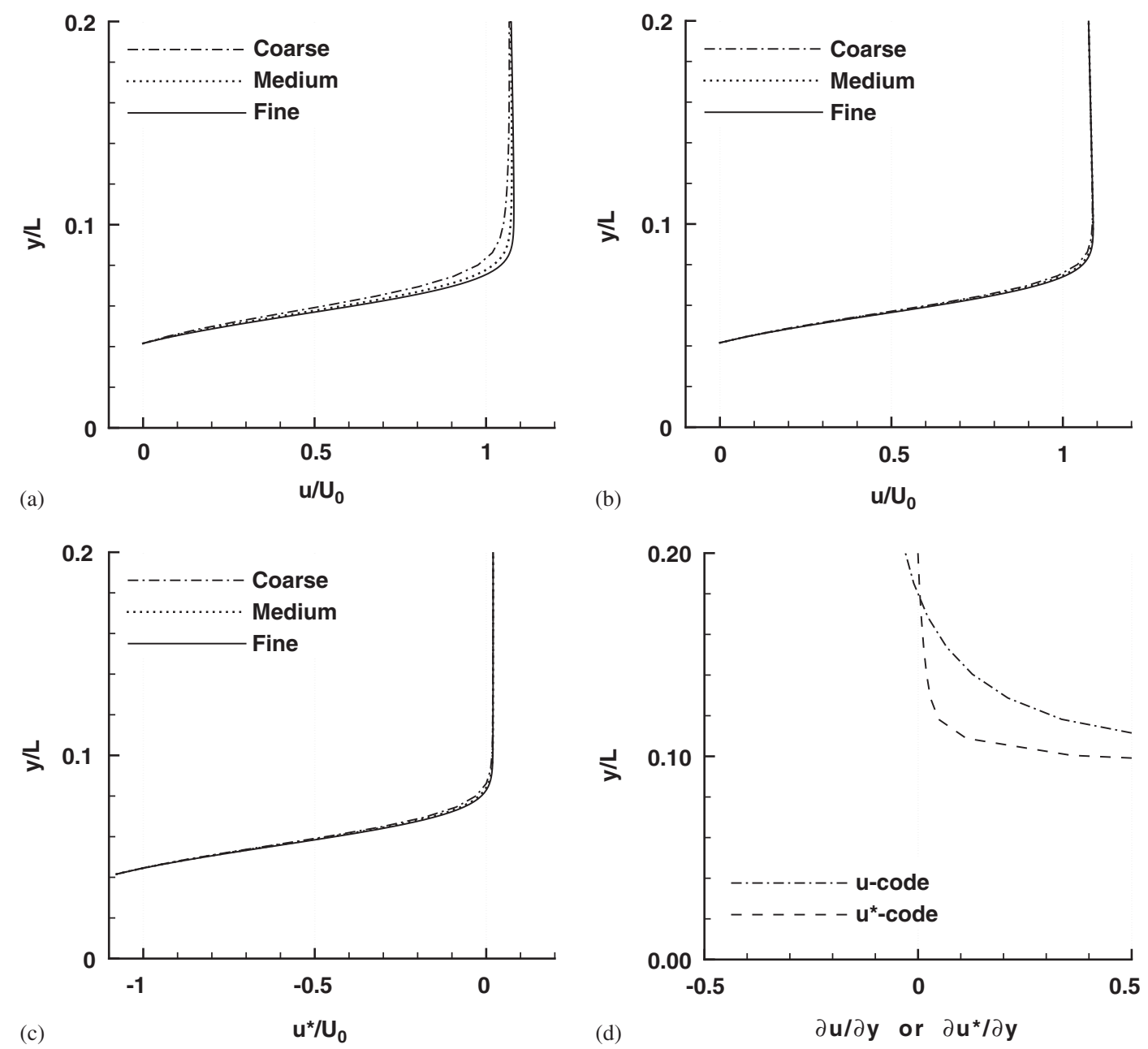

Figure 11. Velocity profiles and their gradients at $x / L=0.54$ (NACA $0010, R e=7900$ ): (a) u-code; (b) $u^{*}$-code; (c) $u^{*}$ profile; and (d) $\partial u / \partial y$ and $\partial u^{*} / \partial y$ in the coarse grid.

where Figure 11(d) shows the gradient of the profiles for the coarse grids corresponding to Figures 11(a) and (c). The second-order central difference scheme is used to compute the gradients. It can be observed that the gradient of the $\mathrm{u}^{*}$-code has smaller values and approaches zero quickly while the gradient of the u-code is larger and becomes negative. This change in the gradient might help the $\mathrm{u}^{*}$-code to reduce the numerical errors caused in the coarse grid.

Figure 12 shows the negative values of pressure coefficient, $-C_{p}\left(=p-p_{0} / \frac{1}{2} \rho U_{0}^{2}\right.$, where $p_{0}$ is the reference pressure) versus $x$. At the leading edge, all three solvers show good agreement, and the stagnation pressure is well captured. At the trailing edge, although the wake source reduces the potential pressure from the stagnation pressure $\left(C_{p}=1\right)$, the potential solver still gives higher pressure than the other two solvers. Nonetheless the $\mathrm{u}^{*}$-code shows good agreement with the u-code. 


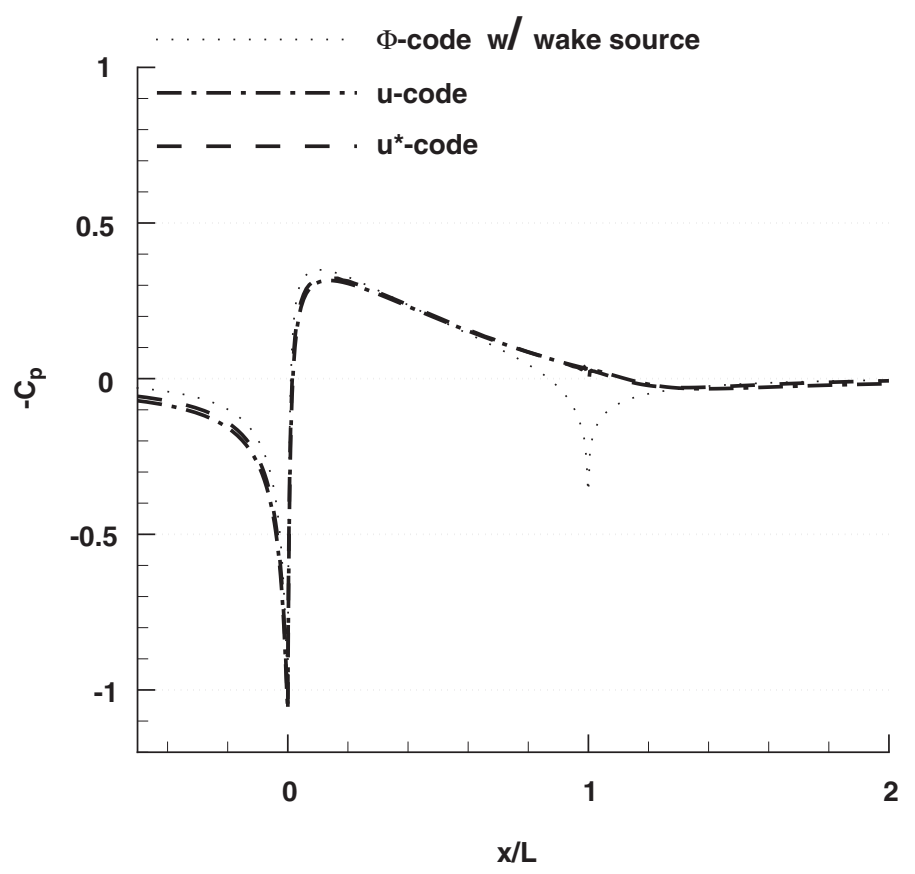

Figure 12. Pressure coefficient (NACA 0010, $R e=7900$ ).

The overall changes from the grid refinement test are shown in Figure 13, where both codes are compared to their corresponding results in the fine grid. Figure 13(a) shows that the $\mathrm{u}^{*}$-code gives considerably smaller differences than the u-code, especially in the coarse grid, where the $\mathrm{u}^{*}$-code gives only about $1 \%$ difference versus the $4 \%$ difference corresponding to the u-code. This difference becomes smaller in the medium grid, where the u-code gives over $1 \%$ and the $\mathrm{u}^{*}$-code gives less than $0.5 \%$. The corresponding CPU times for the various grids are summarized in Figure 13(b). The $\mathrm{u}^{*}$-code in the coarse grid shows a solution as good as or even better than that corresponding to the u-code in the medium grid with a CPU time that is more than 10 times less.

Similar results to those shown in Figure 13 are shown in Figure 14(a) but for the simulations performed with the second-order accurate upwind scheme implemented for the discretization of the convection terms. The results show considerably smaller grid dependency than the results with the first-order upwind scheme. Both codes show less than $1 \%$ difference from the fine grid results as it is clearly shown in Figure 14(b). Compared to the results with the first-order upwind scheme (Figure 13(b)), considerable changes are observed when solving the conventional RANS equations, while very similar results (RMS difference $<0.5 \%$ ) are obtained when solving the complementary RANS equations. The smaller sensitivity to the order of the discretization scheme shown by the proposed solver follows from the already discussed smaller velocity gradients produced by the complementary RANS equations.

Based on the results presented in Figure 13, the adequacy of the grids employed to draw conclusions regarding grid independency is questionable and hence computations are 


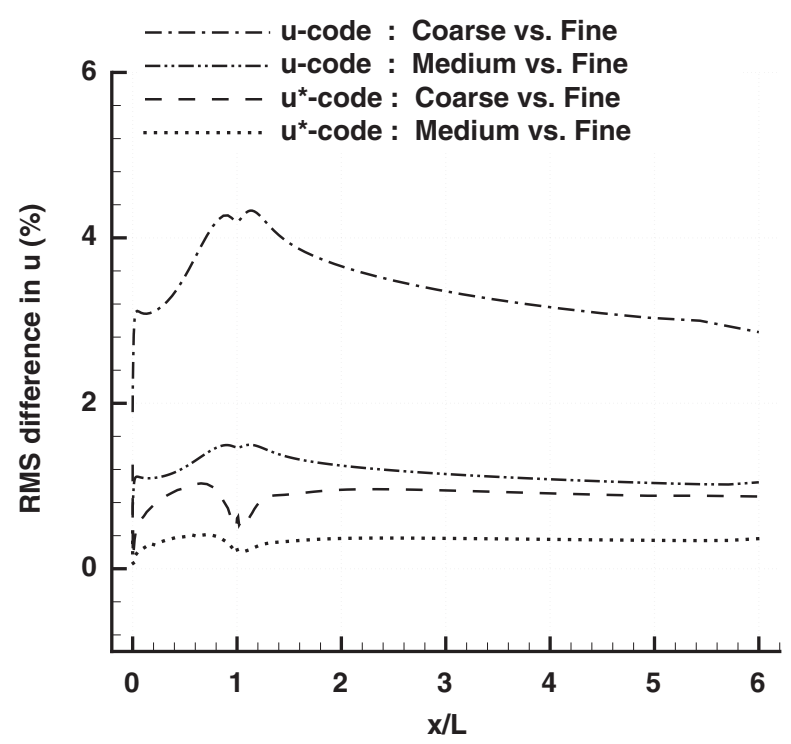

(a)

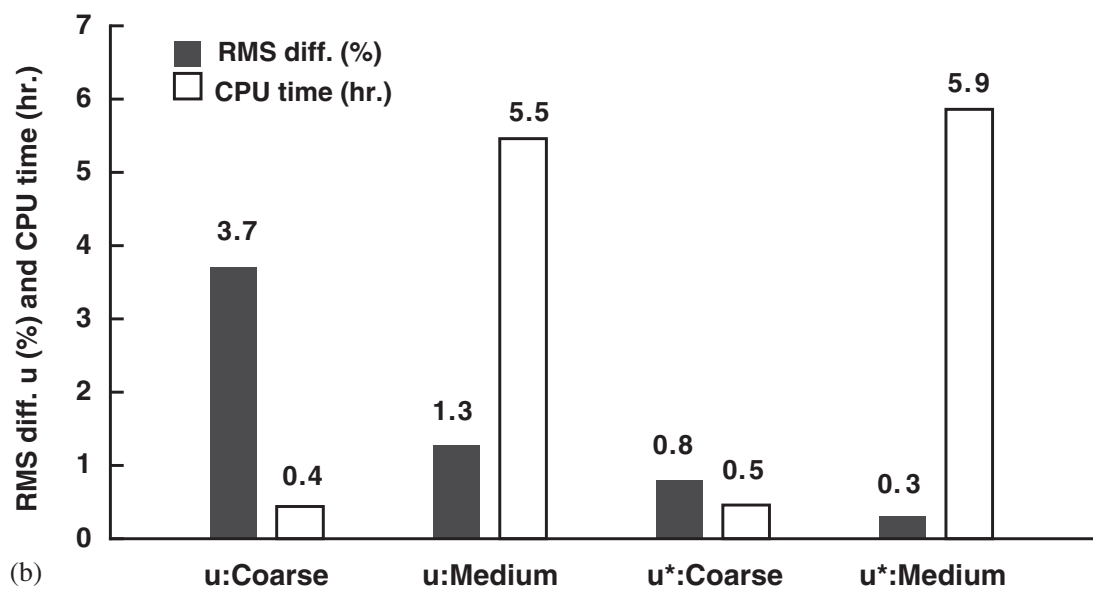

Figure 13. RMS differences and CPU time for various grids (NACA 0010, $R e=7900$ ): (a) RMS difference in the horizontal velocity; and (b) RMS difference and CPU time.

performed with an even coarser grid $(36 \times 21)$, which is denoted as 'very coarse' in the relevant figures.

The corresponding grid dependency results are shown in Figure 14(c). Compared to the results in Figure 14(a), larger differences are observed. Especially, the $\mathrm{u}^{*}$-code in the very coarse grid shows a spike at the trailing edge and large discrepancies in the wake. These discrepancies are shown more clearly in Figures 15(a) and (b), where the detailed distributions of $u^{*}$ and $p^{*}$ along the centreline near the trailing edge are shown. In both results saw-tooth oscillations are observed in the wake. Such oscillations are attributed to the potential solution shown in Figures $15(\mathrm{c})$ and $(\mathrm{d})$, and the fact that the $\mathrm{u}^{*}$-solution is tied to the potential solution trends. 

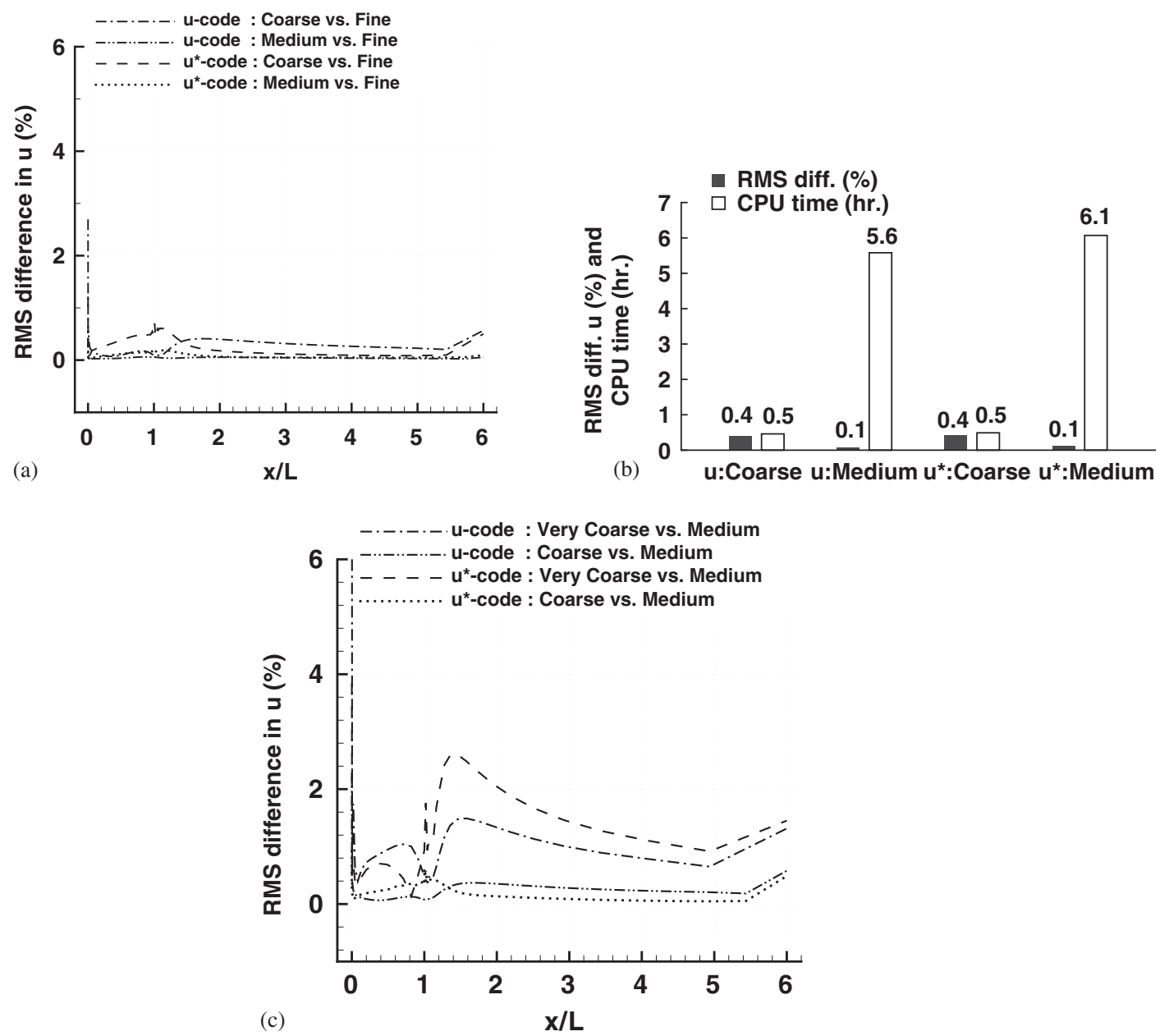

Figure 14. RMS differences and CPU time for various grids; second-order upwind scheme (NACA 0010, $R e=7900)$ : (a) RMS differences in $(71 \times 41),(141 \times 81)$, and $(281 \times 161)$ grids; $(b)$ RMS differences and CPU time in $(71 \times 41),(141 \times 81)$, and $(281 \times 161)$ grids; and $(c)$ RMS differences in $(36 \times 21)$, $(71 \times 41)$, and $(141 \times 81)$ grids.

An attempt is made to overcome the problem just mentioned at the trailing edge by using a smoother potential, that is a potential solution with a smooth velocity profile at the trailing edge. To accomplish this, a fairing technique is employed near the trailing edge region, and to do so the locations of the control and source points are changed as shown in Figure 16(a). A detailed distribution of both control and source points at the leading and the trailing edges is shown in Figures 16(b) and (c). As shown in Figure 16(b), the leading edge has two control points; one for the tangential direction and the other one for the normal direction. These two control points and corresponding sources constitute a stagnation point at the leading edge. At the trailing edge a fairing technique is used to reduce the peak shown earlier for 

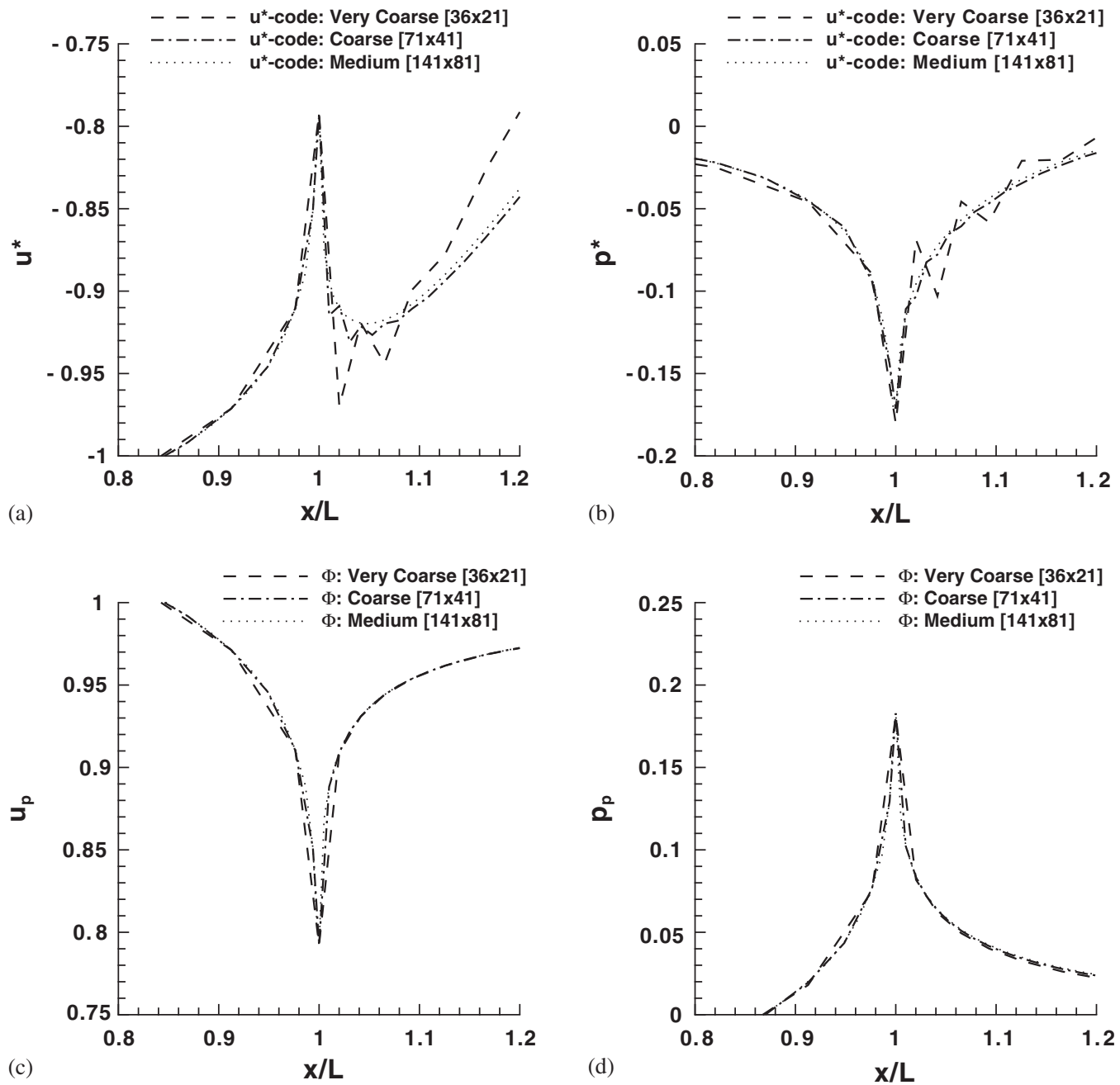

Figure 15. Velocity and pressure distribution near the trailing edge along the centreline (NACA 0010, $R e=7900$ ): (a) $u^{*}$; (b) $p^{*}$; (c) $u_{p}$; and (d) $p_{\mathrm{p}}$.

$u_{p}$ and $p_{\mathrm{p}}$ (see Figures $15(\mathrm{c})$ and $(\mathrm{d})$ ). This is accomplished by fairing the body with a circular arc whose size is controlled by a parameter, $L_{\text {tail }}$, which is the length from the trailing edge to the end of the arc. The same body boundary condition is used on the faired body surface as used on the actual body surface. The location of the sources is also determined by the same method as used in the previous potential. On the centreline upstream and downstream of the body the condition, $v_{p}=0$, is applied to ensure the symmetry of the potential solution.

By employing this fairing technique, the peaks in $u_{p}$ and $p_{\mathrm{p}}$ are smoothed out considerably as shown in Figures 17(a) and (b). Figures 17(c) and (d) shows that the faired trailing edge, 


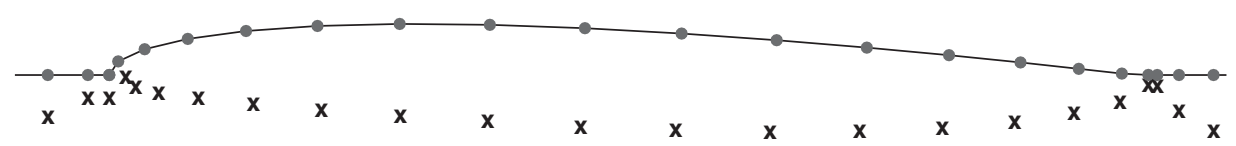

(a)
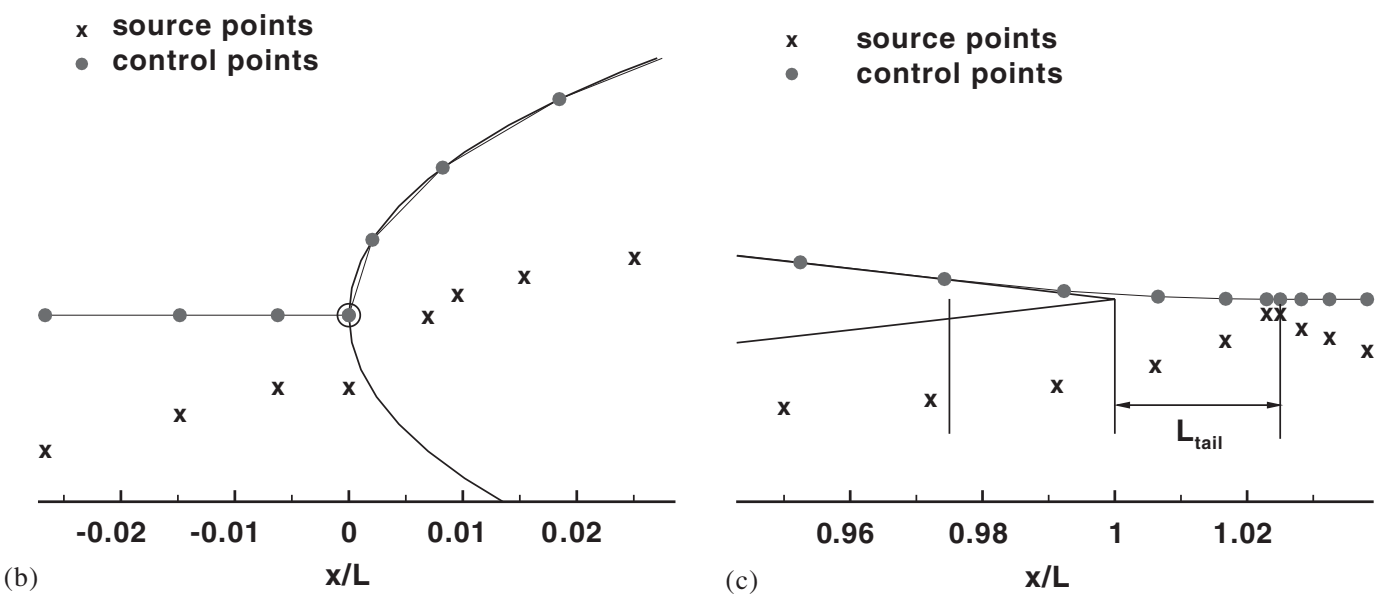

Figure 16. Modified distribution of control points and source points (NACA 0010, $R e=7900$ ):

(a) control and source points; (b) near LE; and (c) near TE.

in fact, has reduced the numerical errors in the very coarse grid in the wake by as much as $1 \%$ of the free stream. Compared to Figures 15(a) and (b), Figures 17(c) and (d) show that the oscillations are visibly reduced and the discrepancies in the very coarse grid are significantly reduced.

The comparison of $-C_{p}$ between the potential, the u-code and the $\mathrm{u}^{*}$-code is shown in Figure 18. As shown, the sharp peak in the potential shown in the previous result is smoothed in the new result.

The comparison of the grid dependency between the $\mathrm{u}^{*}$-code and the u-code is shown in Figure 19(a). As mentioned, the difference between the very coarse grid result and the medium grid result in the $\mathrm{u}^{*}$-code is now less than the corresponding result in the u-code. This result shows that the smoothness of a potential solution is critically important in the solution of the complementary RANS equations. The overall RMS differences in $u$ between the solutions and their CPU times are shown in Figure 19(b). Although the difference of the $\mathrm{u}^{*}$-code between the very coarse grid and the medium grid $(1.1 \%)$ is not as good as the corresponding difference of the $\mathrm{u}$-code in the coarse grid $(0.5 \%)$, the $\mathrm{u}^{*}$-code gives a difference that is twice as small as the $\mathrm{u}$-code in the very coarse grid $(2.2 \%)$.

4.2.2. Turbulent flow over NACA0012 airfoil. For turbulent flow computations, a NACA 0012 airfoil is employed instead of NACA 0010 because of the availability of experimental and other numerical data for this case. Detailed comparisons of the numerical results obtained with the complementary RANS equations and those from the conventional RANS solver, the numerical data of Rhie and Chow [38] and the experimental results of Gregory and 

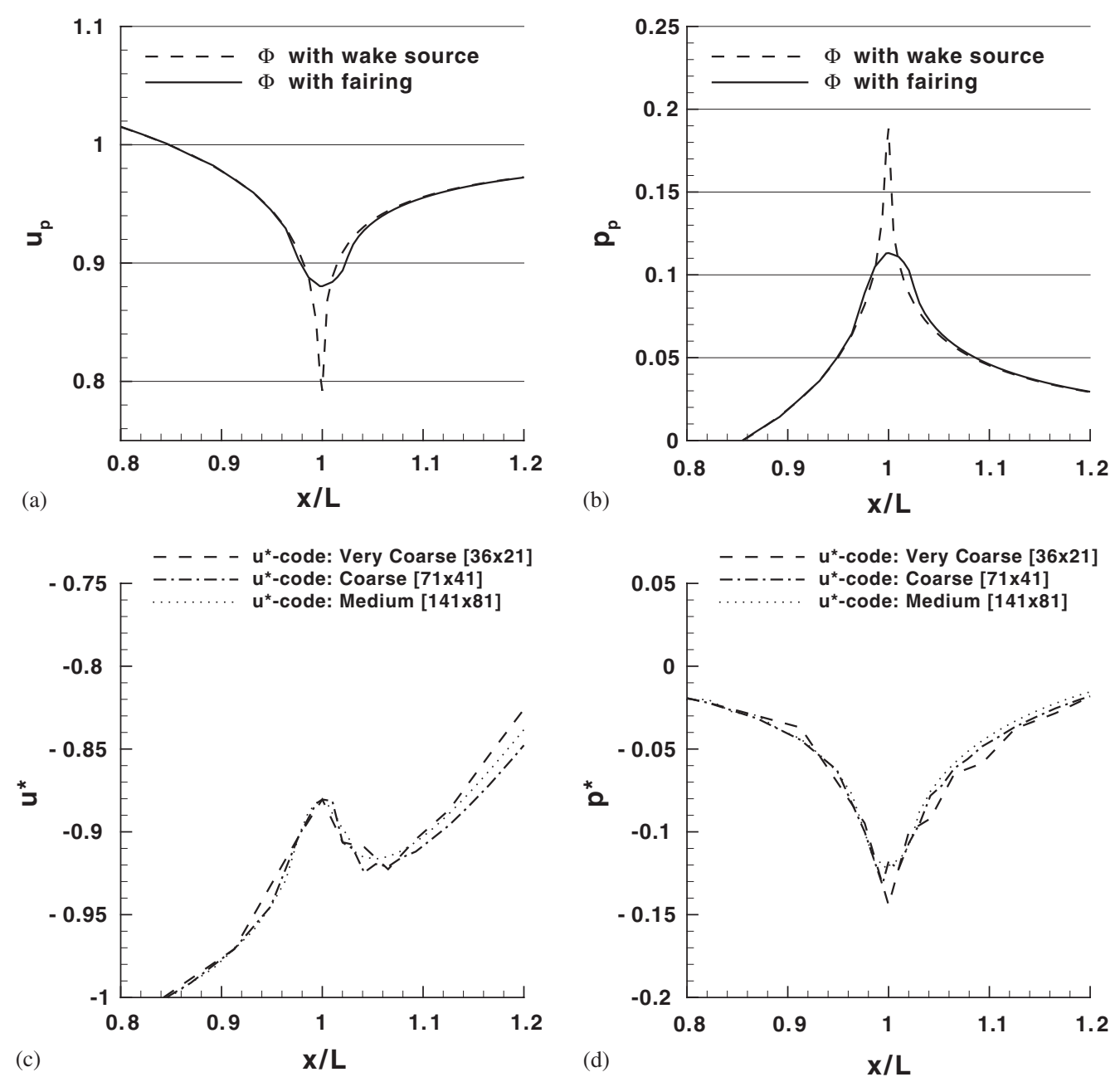

Figure 17. Velocity and pressure distribution near the trailing edge along the centreline with a faired trailing edge (NACA 0010, $R e=7900$ ): (a) $u_{p}$; (b) $p_{\mathrm{p}}$; (c) $u^{*}$; and (d) $p^{*}$.

O'Reilly [39] at $R e=2.8 \times 10^{6}$ can be found in Kim [40] and Kim et al. [41]. Here emphasis will be given to further corroborate the ability of the solver to give accurate results in coarser grids for turbulent flows.

In the solution of the complementary RANS equations the potential with the faired trailing edge is used and the second-order upwind discretization scheme is employed for the convection terms. Similar grid generation algorithms are employed and the boundary conditions can be inferred from the previous sections. The following grids, $(141 \times 81),(71 \times 41)$, and $(36 \times 22)$ are used to perform the grid independence study.

The overall RMS differences in the horizontal velocity between the coarse, the medium and the fine grids are shown in Figure 20 along with the corresponding CPU times. The trends 


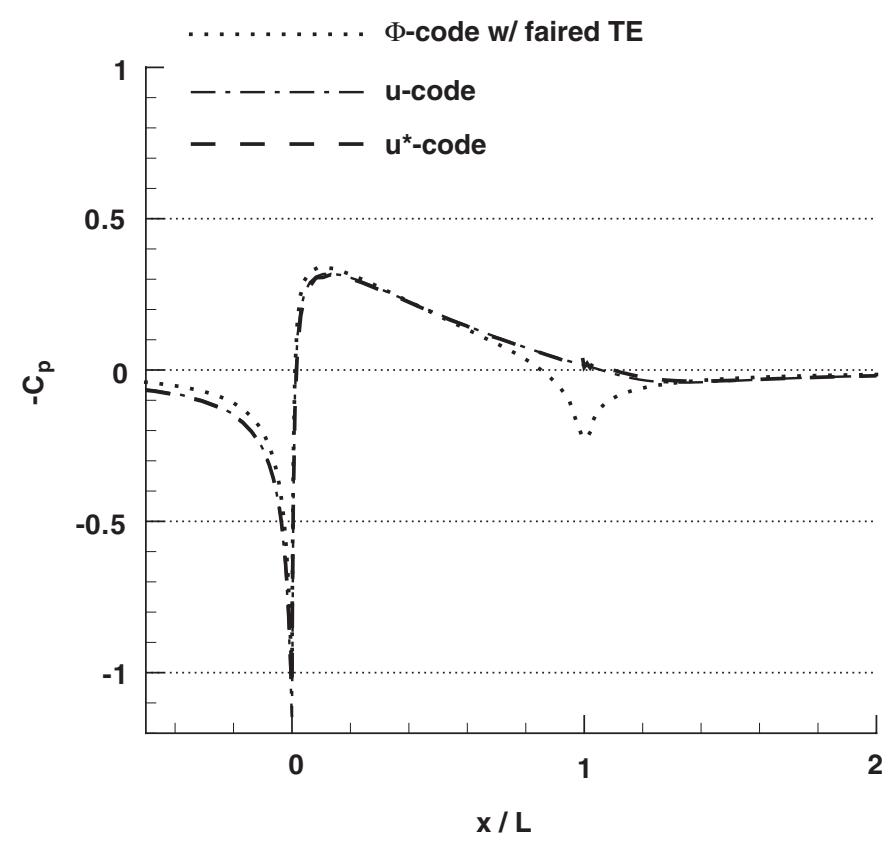

Figure 18. Pressure coefficient; with the smoothed potential and second-order upwind scheme (NACA 0010, $R e=7900$ ).

are similar to those found for the laminar case. In other words, the $\mathrm{u}^{*}$-code computations in the coarse and the fine grids shows less differences than the corresponding u-code results. In the laminar case, the RMS difference in the $\mathrm{u}^{*}$-code $(1.1 \%)$ is about $50 \%$ of the RMS difference in the u-code $(2.2 \%)$. In the turbulent case, the RMS difference in the $\mathrm{u}^{*}$-code $(0.7 \%)$ is reduced to only about $30 \%$ of the RMS difference in the u-code $(2.2 \%)$. However this result is to be expected due to the reduction of the region where viscous effects are important. Compared to the laminar results shown in Figure 19, Figure 20(a) shows that the differences are generally smaller over the body in the turbulent computations, except near the trailing edge where the $\mathrm{u}^{*}$-code shows slightly larger differences than the u-code.

\section{CONCLUSIONS}

By decomposing a velocity field into an arbitrary potential velocity vector and the remaining velocity vector a new set of RANS equations, called complementary RANS equations are derived. In the complementary RANS solver the chosen potential solution coexists with a viscous solution. The equations have been validated for steady laminar and turbulent flows, specifically flow in a square duct $(R e=790)$ (results not shown herein), over a flat plate $\left(R e=7900\right.$ and $\left.1.6 \times 10^{6}\right)$ and over a NACA airfoil $\left(R e=7900\right.$ and $\left.2.8 \times 10^{6}\right)$.

The results from the complementary RANS solver show good agreement with the conventional RANS solver. The most interesting result is that by introducing the potential solution in 

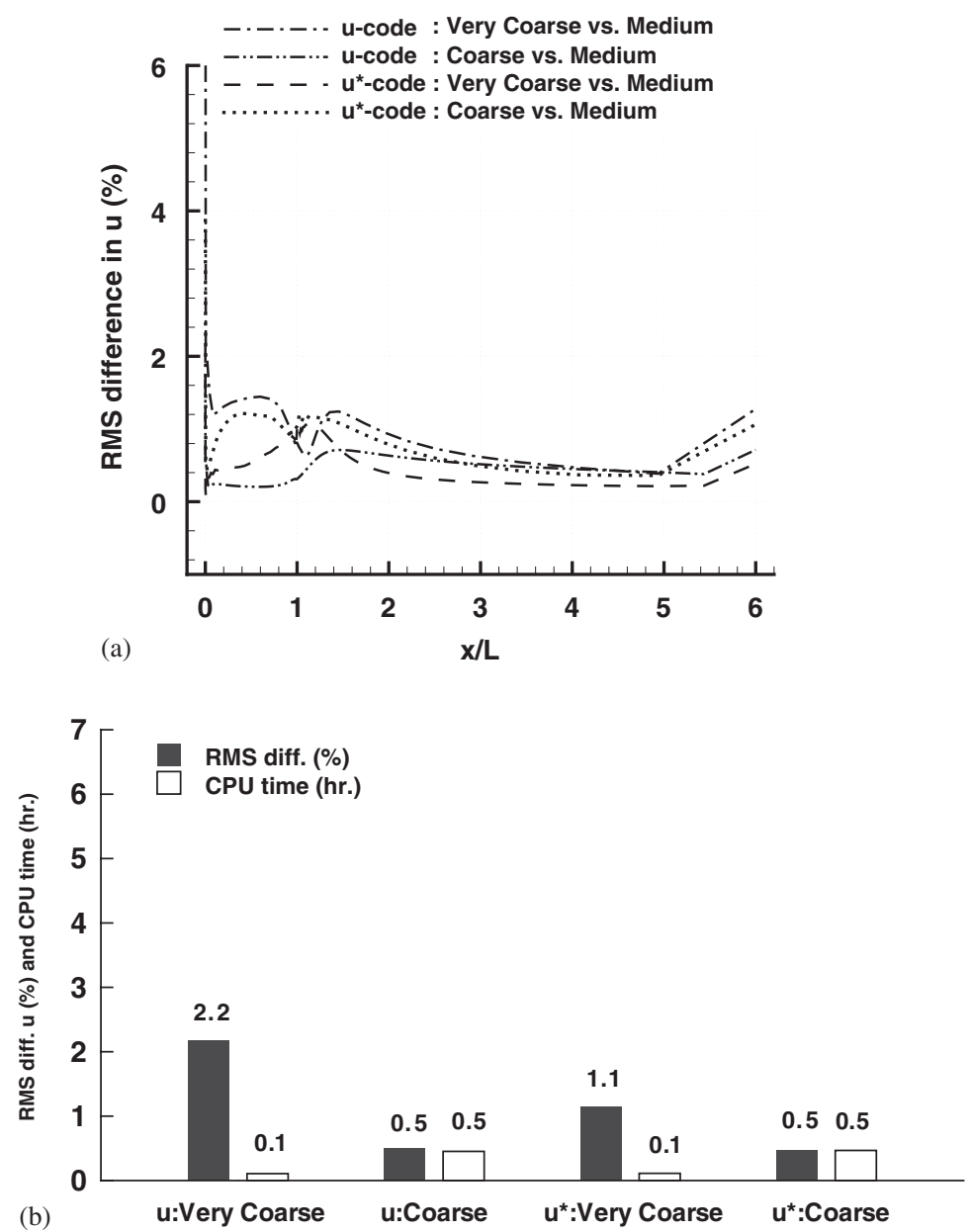

Figure 19. RMS differences; second-order upwind scheme in very coarse grid $(36 \times 21)$, coarse grid $(71 \times 41)$, and medium grid $(141 \times 81)($ NACA $0010, R e=7900)$ : (a) RMS differences in the horizontal velocity; and (b) RMS differences and CPU time.

the RANS solver, the gradients of the velocity profiles corresponding to the complementary velocity may be significantly smaller. This in turn translates into the need of less resolved grids in order to obtain the same accuracy that required by the conventional RANS solver. This also has a repercussion on the order of accuracy of the discretization schemes used. It is shown that a first-order upwind scheme for the convection terms in the complementary RANS solver can give as accurate results as a second-order scheme in the conventional RANS solver. The corresponding computational time savings are also documented in this study.

A discussion is also presented to assess the impact of the arbitrarily chosen potential solution on the overall computations. It is shown that the smoothness of the gradient of the potential solution plays an important role in the proposed numerical solver. While not shown here it is speculated that a careful choice of the potential solution would contribute 

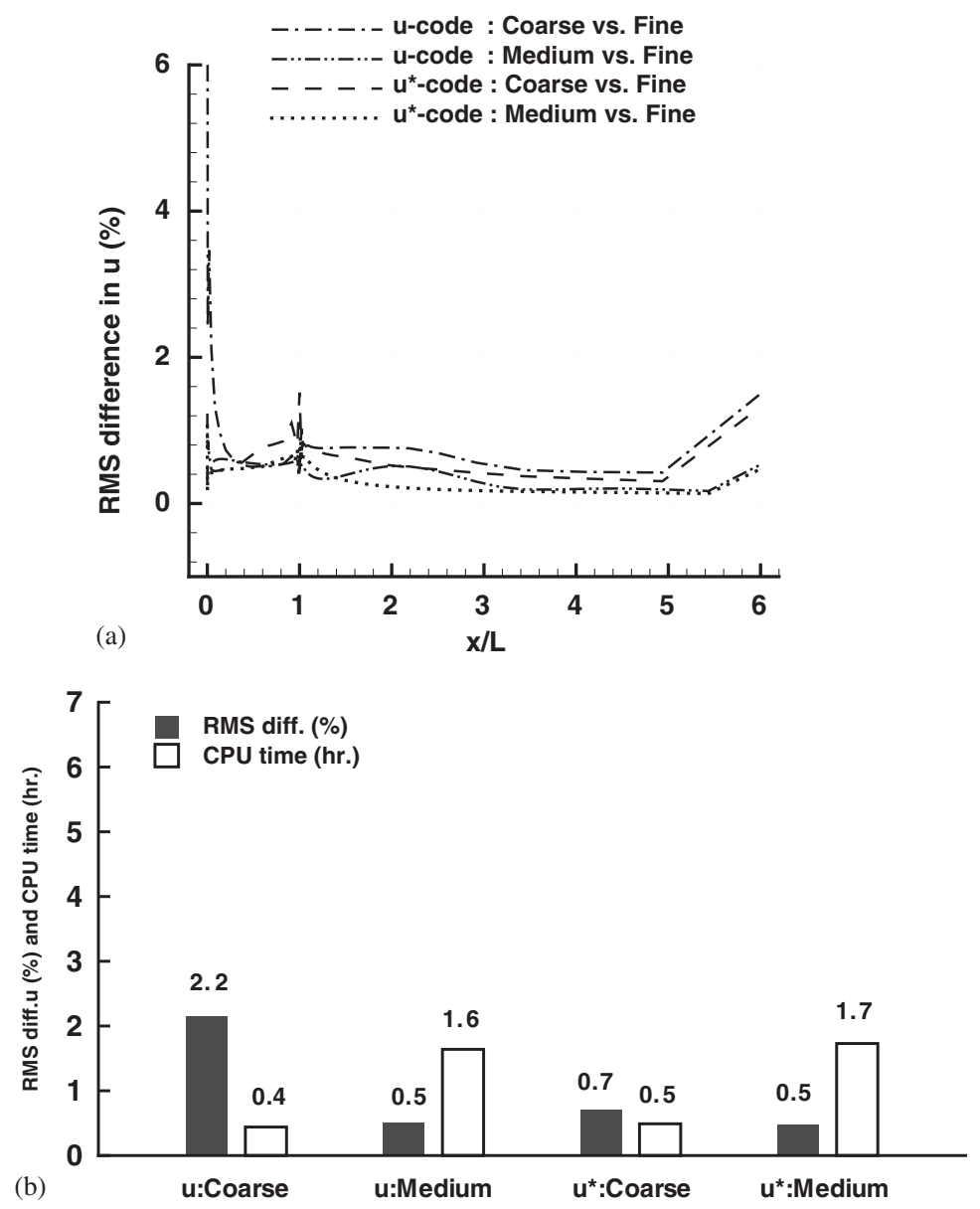

Figure 20. RMS differences in coarse grid $(36 \times 22)$, medium grid $(71 \times 41)$, and fine grid $(141 \times 80)\left(\right.$ NACA $\left.0012, R e=2.8 \times 10^{6}\right)$ : (a) RMS differences in horizontal velocity; and (b) RMS differences and CPU time.

to a faster decay of the viscous solution in the far field, which could possibly translate into smaller computational domains.

\section{ACKNOWLEDGEMENTS}

This research was partially supported by the Office of Naval Research Grant Number N00014-01-2-0008, with Dr Patrick Purtell as ONR Program Manager. This support is gratefully acknowledged.

\section{REFERENCES}

1. Prandtl L. Über flüssigkeitsbewegung bei sehr kleiner reibung. Proceedings of the Third International Mathematical Congress, Heidelberg, 1904; 484-494 [English Translation in NACA Technical Memo. 452]. 
2. Lighthill MJ. On displacement thickness. Journal of Fluid Mechanics 1958; 4(4):383-392.

3. Lock RC, Williams BR. Viscous-inviscid interactions in external aerodynamics. Progress in Aerospace Sciences 1987; 24(2):51-171.

4. Miyata H, Zhu M, Watanabe O. Numerical study on a viscous flow with free-surface waves about a ship in steady straight course by a finite-volume method. Journal of Ship Research 1992; 36(4):332-345.

5. Farmer J, Martinelli L, Jameson A. Fast multigrid for solving incompressible hydrodynamic problems with free surfaces. AIAA Journal 1994; 32(6):1175-1182.

6. Tahara Y, Stern F. A large-domain approach for calculating ship boundary layers and wakes for nonzero Froude number. Proceedings of the CFD Workshop Tokyo 1994; 1:45-55.

7. Stern F, Yoo SY, Patel VC. Interactive and large-domain solutions of higher-order viscous-flow equations. $A I A A$ Journal 1988; 26(9):1052-1060.

8. Tahara Y, Stern F, Rosen B. An interactive approach for calculating ship boundary layers and wakes for nonzero Froude number. Journal of Computational Physics 1992; 98:33-53.

9. Villeger F, Allessandrini B. Interaction between free surface flow and boundary layer around a ship model. Proceedings of the 7th International Workshops on Water Waves and Floating Bodies, France, 1992; 291-295.

10. Chen HC, Lin WM, Weems KM. Interactive zonal approach for ship flows including viscous and nonlinear wave effects. Proceedings of the 6th International Conference on Numerical Ship Hydrodynamics, Iowa City, U.S.A., 1993; 341-363.

11. Campana E, Di Mascio A, Esposito PG, Lalli F. Domain decomposition in free surface viscous flows. Proceedings of the 6th International Conference on Numerical Ship Hydrodynamics, Iowa City, U.S.A., 1993; 329-340.

12. Campana E, Di Mascio A, Esposito PG, Lalli F. Viscous-inviscid coupling in free surface ship flows. International Journal for Numerical Methods in Fluids 1995; 21:699-722.

13. Chen HC, Lee SK. Interactive RANS/Laplace method for nonlinear free surface flows. Journal of Engineering Mechanics 1996; 122(2):153-162.

14. Chen HC, Lee SK. RANS/Laplace calculations of nonlinear waves induced by surface-piercing bodies. Journal of Engineering Mechanics 1999; 125(11):1231-1242.

15. Dommermuth D, Innis G, Luth T, Novikov E, Schlageter E, Talcott J. Numerical simulation of bow waves. Proceedings of the 22nd Symposium on Naval Hydrodynamics, Washington, DC, U.S.A., 1998; 508-521.

16. Ferrant P, Gentaz L, Alessandrini B, Le Touzé D. A potential/RANSE approach for regular water wave diffraction about 2-d structures. Ship Technology Research 2003; 50:165-171.

17. Helmholtz HLF. Über integrale der hydrodynamischen gleichungen, welch den wirbelbewegungen entsprechen. Crelles Journal 1858; 55:25-55.

18. Chen HC, Patel VC. Calculation of trailing-edge, stern and wake flows by a time-marching solution of the partially-parabolic equations. IIHR Report 285. Iowa Institute of Hydraulic Research, University of Iowa, U.S.A., 1985.

19. Chen HC, Patel VC. Neal-wall turbulence models for complex flows including separation. AIAA Journal 1988; 26(6):641-648.

20. Beck RF. Time-domain computations for floating bodies. Applied Ocean Research 1994; 16:267-282.

21. Patel VC, Sotiropoulos F. Longitudinal curvature effects in turbulent boundary layers. Progress in Aerospace Sciences 1997; 33:1-70.

22. Sinha SK, Sotiropoulos F, Odgaard AJ. Three-dimensional numerical model for flows through natural rivers. Journal of Hydraulic Engineering 1998; 124(1):13-24.

23. Vanka SP. Second-order upwind differencing in a recirculating flow. AIAA Journal 1987; 25(11):1435-1441.

24. Chorin AJ. Numerical solution of the Navier-Stokes equations. Mathematics of Computation 1968; 22: $745-762$.

25. Beam RM, Warming RF. An implicit factored scheme for the compressible Navier-Stokes equations. AIAA Journal 1978; 16(4):393-402.

26. Hoffmann KA, Chiang ST. Computational Fluid Dynamics for Engineers. Engineering Education System: Whichita, 1993.

27. Sotiropoulos F, Abdallah S. Coupled fully implicit solution procedure for the steady incompressible NavierStokes equations. Journal of Computational Physics 1990; 87:328-348.

28. Kim K, Beck RF, Sirviente AI. A viscous-inviscid interaction study using complementary RANS equations. Proceedings of the 8th International Conference on Numerical Ship Hydrodynamics, Busan, Korea, 2003; 2:248-260.

29. White FM. Viscous Fluid Flow. McGraw-Hill: New York, 1991.

30. Humphrey JAC, Taylor AMK, Whitelaw JH. Laminar flow in a square duct of strong curvature. Journal of Fluid Mechanics 1977; 83(3):509-527.

31. Thomson JF, Warsi ZUA, Mastin CW. Numerical Grid Generation. Elsevier: New York, 1985.

32. Blasius H. Grenzschichten in flüssikeiten mit kleiner reibung. Zeitschrift für Angewandte Mathematic und Physik 1908; 56:1-37 [English translation in NACA Technical Memo. 1256]. 
33. DeGraaff DB, Eaton JK. Reynolds-number scaling of the flat-plate turbulent boundary layer. Journal of Fluid Mechanics 2000; 422:319-346.

34. Ramaprian BR, Patel VC, Sastry MS. The symmetric turbulent wake of a flat plate. AIAA Journal 1982; 20: $1228-1235$.

35. Kim J, Moin P, Moser R. Turbulent statistics in fully developed channel flow at low Reynolds number. Journal of Fluid Mechanics 1987; 177:133-166.

36. Coles DE. The turbulent boundary layer in a compressible fluid. Technical Report R-403-PR, United States Air Force Project RAND, 1962.

37. Cao Y, Schultz WW, Beck RF. Three-dimensional desingularized boundary integral methods for potential problems. International Journal for Numerical Methods in Fluids 1991; 12:785-803.

38. Rhie CM, Chow WL. Numerical study of the turbulent flow past an airfoil with trailing edge separation. AIAA Journal 1983; 21(11):1525-1532.

39. Gregory N, O'Reilly CL. Low speed aerodynamic characteristics of NACA 0012 airfoil section, including the effects of upper surface roughness simulation Hoarfrost. Aerosol Report, National Physical Laboratory, Teddington, England. 1970; 1308.

40. Kim K. A viscous-inviscid interaction study using complementary RANS equations. Ph.D. Thesis, University of Michigan, 2004.

41. Kim K, Sirviente AI, Beck RF. Complementary RANS equations for viscous flow computations. Proceedings of the 25th Symposium on Naval Hydrodynamics, Newfoundland, Canada, 2004. 